- Estudi -

\title{
LAS ENTIDADES SUB-NACIONALES EN NORTEAMÉRICA Y LA LUCHA CONTRA EL CAMBIO CLIMÁTICO: DESARROLLO NORMATIVO Y VINCULACIÓN DE SISTEMAS DE COMERCIO DE DERECHOS DE EMISIÓN ${ }^{1}$
}

\author{
MAR CAMPINS ERITJA \\ Profesor Titular de Derecho Internacional Público (Derecho Comunitario Europeo) \\ Universitat de Barcelona \\ mcampins@ub.edu
}

Recibido: 15 de noviembre de 2010 / Aceptado: 17 de diciembre de 2010

RESUMEN: Mientras que en Europa la interdependencia y la dimensión transfronteriza de las cuestiones ligadas al cambio climático ha facilitado una cierta "continentalización" de la gestión de este fenómeno, favorecida por el carácter intergubernamental de las medidas que se adoptan en el marco de la Unión Europea, al otro lado del Atlántico la transversalidad de este mismo fenómeno explica la necesidad de que, ante las limitaciones del marco regional, se pongan en marcha mecanismos que faciliten la intervención no sólo estatal sino también de las entidades sub-nacionales. En este sentido, la ausencia de la acción federal tanto en Estados Unidos como en Canadá ha comportado un mayor desarrollo de la acción de las entidades sub-nacionales, que han tomado el liderazgo en la lucha contra el cambio climático. Son estas medidas las que se han visualizado en el escenario internacional. Ello ha favorecido el establecimiento de mecanismos de coordinación de la acción de estas entidades sub-

\footnotetext{
1 Este trabajo forma parte de un estudio más amplio realizado gracias a la concesión de una beca del Institut d'Estudis Autonòmics para la investigación sobre las autonomías políticas locales, desarrollada en la Université de Montréal, Quebec, Canadá, durante el curso 2009-2010. (Resolución IRP/2009, de 18 de Mayo de 2009). Por otra parte, se enmarca en el proyecto de investigación del Ministerio de Educación y Ciencia titulado "El derecho ambiental como modelo para la transformación de la actividad de los poderes públicos" (Referencia SEJ 2007/61972/JURI)."
} 
nacionales en el seno de redes transnacionales, que han ido adquiriendo una mayor relevancia en la implementación del Convenio Marco sobre el Cambio Climático y del Protocolo de Kyoto, particularmente en relación a uno de sus instrumentos, el comercio de los derechos de emisión de gases de efecto invernadero. En este contexto, la futura vinculación de los sistemas de comercio de derechos de emisión de gases de efecto invernadero en Norteamérica con el sistema de la Unión Europea presenta diversos retos de carácter material y formal.

RESUM: Mentre que a Europa la interdependència i la dimensió transfronterera de les qüestions vinculades al canvi climàtic ha facilitat una certa "continentalització" de la gestió d'aquest fenomen, afavorida pel caràcter intergovernamental de les mesures que s'adopten en el marc de la Unió Europea, a l'altre costat de l'Atlàntic la transversalitat d'aquest mateix fenomen explica la necessitat que, davant les limitacions del marc regional, es posin en marxa mecanismes que facilitin la intervenció no només estatal sinó també de les entitats sub-nacionals. En aquest sentit, l'absència d'acció federal tant als Estats Units com a Canadà ha comportat un major desenvolupament de l'acció de les entitats sub-nacionals, que han pres el lideratge en la lluita contra el canvi climàtic. Aquestes mesures són les que s'han visualitzat en l'escenari internacional. Això ha afavorit l'establiment de mecanismes de coordinació de l'acció d'aquestes entitats subnacionals en el si de xarxes transnacionals, que han anat adquirint una major rellevància en la implementació del Conveni Marc sobre el Canvi Climàtic i del Protocol de Kyoto, particularment en relació a un dels seus instruments, el comerç dels drets d'emissió de gasos d'efecte hivernacle. En aquest context, la futura vinculació dels sistemes de comerç de drets d'emissió de gasos d'efecte hivernacle a Amèrica del Nord amb el sistema de la Unió Europea presenta diversos reptes de caràcter material i formal.

ABSTRACT: In Europe, the interdependence and cross-border dimension of climate change's issues as well as the intergovernmental nature of the European Union measures, have favored certain "continentalization" of its management. In North America, the transversal character of these same issues and the limitations of regional framework have favored the intervention of the States as well as the sub-national 
bodies. The lack of federal action in United States and Canada implies a further development of sub-national's action. Sub-national bodies have taken the lead fighting the climate change and they have acquired a relevant presence at international level, too. This has encouraged the establishment of sub-national bodies' coordination within transnational networks, which have gained a larger role in the implementation of the Framework Convention on Climate Change and the Kyoto Protocol, especially in what concerns the greenhouse gases emission trading. In this context, the future linking of these emission trading systems with European Union emission trading system presents several challenges from the material and formal perspectives.

PALABRAS CLAVE: Cambio climático - mercado de derechos de emisión de gases de efecto invernadero - entidades sub-nacionales - Unión Europea - Canadá Estados Unidos.

PARAULES CLAU: Canvi climàtic - mercat de drets d'emissió de gasos d'efecte hivernacle - entitats subnacionals — Unió Europea — Canadà — Estats Units

KEYWORDS: Climate change — greenhouse gas emission rights market — subnational entities — European Union — Canada — United States

Sumario: I. Introducción. II. La pérdida de influencia del legislador federal en la lucha contra el cambio climático en Norteamérica. 1. Una intervención poco exitosa del poder federal en Estados Unidos. 2. El inmovilismo del poder federal en Canadá. III. El dinamismo de las entidades sub-nacionales en la lucha contra el cambio climático en Norteamérica. 1. Una intervención de los estados basada en la cooperación entre administraciones en Estados Unidos. 2. Una intervención de las provincias basada en la exclusividad de la competencia en Canadá. 3. La acción de las entidades sub-nacionales y la articulación de redes transnacionales en la lucha contra el cambio climático en Norteamérica. 3.1.Regional Greenhouse Gas Initiative. 3.2.Western Climate Initiative. 3.3.Midwestern Regional Greenhouse Gas Reduction Accord. IV. Un caso singular de intervención de las entidades sub-nacionales: la vinculación de los sistemas de comercio de emisiones norteamericanos con el sistema de comercio de emisiones de la Unión Europea. 1. Las limitaciones materiales a la conclusión de acuerdos de vinculación de los sistemas de comercio de derechos de emisión. 2. Las limitaciones formales a la conclusión de acuerdos de vinculación de los sistemas de comercio de derechos de emisión. V. Observaciones finales.

\section{INTRODUCCIÓN}


Mientras que en Europa la interdependencia y la dimensión transfronteriza de las cuestiones ligadas al cambio climático ha facilitado una cierta "continentalización" de la gestión de este fenómeno, favorecida por el carácter intergubernamental de las medidas que se adoptan en el marco de la Unión Europea (en adelante UE), al otro lado del Atlántico la transversalidad de este mismo fenómeno explica la necesidad de que, ante las limitaciones del marco supra regional, se pongan en marcha mecanismos que faciliten la intervención no sólo estatal sino también de las entidades sub-nacionales. En este sentido, la ausencia de la acción federal tanto en Estados Unidos como en Canadá ha comportado un mayor desarrollo de la acción de las entidades sub-nacionales, que han tomado el liderazgo en la lucha contra el cambio climático. Ello ha favorecido el establecimiento de mecanismos de coordinación de la acción de estas entidades subnacionales en el seno de redes transnacionales, que han ido adquiriendo una mayor relevancia en la implementación del Convenio Marco sobre el Cambio Climático (en adelante CMCC) y del Protocolo de Kyoto (en adelante PK), particularmente en relación a uno de sus instrumentos, el comercio de los derechos de emisión de gases de efecto invernadero. En este contexto, la futura vinculación de los sistemas de comercio de derechos de emisión de gases de efecto invernadero en Norteamérica con el sistema de la UE presenta diversos retos de carácter material y formal.

Sistemáticamente, y a los efectos de su exposición, se ha organizado este trabajo en torno a tres ejes. En el primer apartado se aborda el examen de las propuestas que han surgido de la administración federal a los Estados Unidos y en Canadá, que se caracterizan, dentro de un inmovilismo general, por el relativo liderazgo del primero al nivel de propuestas y por la pérdida de influencia de los segundo en Norteamérica. El segundo apartado analiza como en el contexto norteamericano y ante la inactividad federal han sido las entidades sub-nacionales las que han propiciado los principales desarrollos normativos en materia de lucha contra el cambio climático, lo que se ve favorecido por una amplia descentralización en esta materia. En este apartado se examina también el desarrollo, en este mismo ámbito, de mecanismos de coordinación de las entidades sub-nacionales en la forma de redes transnacionales, que han adquirido un papel cada vez más relevante en el derecho internacional. El estudio se cierra con un tercer apartado en el que se aborda un supuesto especial de intervención de las entidades 
sub-nacionales, como es la vinculación de los sistemas de comercio de emisiones en Norteamérica con el sistema de comercio de emisiones de la UE, un aspecto previsto de manera expresa a la Directiva 2009/29/CE. Finalmente, al último apartado se apuntan algunas observaciones a guisa de conclusión.

\section{LA PÉRDIDA DE INFLUENCIA DEL LEGISLADOR FEDERAL EN LA LUCHA CONTRA EL CAMBIO CLIMÁTICO EN NORTEAMÉRICA}

\section{Una intervención poco exitosa del poder federal en Estados Unidos}

Desde la toma de posesión el 20 de Enero de 2009 del Presidente Obama, la intervención de la administración federal en materia de protección del medio ambiente ha sido, en Estados Unidos, la más relevante de los últimos diez años. Sin embargo, a diferencia de lo que ocurre en otros ámbitos como la defensa nacional, la política monetaria, la política exterior o el establecimiento de aranceles, la Constitución de Estados Unidos no dispone en esta materia la existencia de una competencia federal expresa. Por esa razón, como cualquier otro poder no delegado expresamente en virtud de la Constitución, éste estaría en principio reservado a los estados.

No obstante, la intervención federal se ha venido produciendo sobre la base constitucional que ofrece el apartado tercero de sección 8 del artículo I de la Constitución, lo que se conoce como la "cláusula de comercio interestatal", cláusula que no ha estado exenta de cierta controversia jurisprudencial ${ }^{2}$ a lo largo de la historia constitucional de Estados Unidos.

La noción de comercio inter-estatal, conforme a la tradición constitucional norteamericana ${ }^{3}$, comprende todo movimiento de personas y bienes a través de las fronteras estatales, así como toda comunicación o transmisión de conocimientos y toda

\footnotetext{
2 Vid. CASTELLA ANDREU, J.M., "Tribunal Supremo y cambios en el federalismo de Estados Unidos", en Teoría y realidad constitucional, núm. 24, 2009, p. 491, p. 496 y ss.

3 Sentencias de la Corte Suprema de 19.4.1920, United States v. Simpson, 252 U.S. 465 (1920); de 15.1.1917, Caminetti v. United States, 242 U.S. 470 (1917); de 5.6.1944, United States v. South-Eastern Underwriters Ass'n, 322 U.S. 533; en Vlex Estados Unidos, Constitution of the United States (Annotated), recuperado el 25 de diciembre 2010 de http://vlex.com/vid/section-powers-ofcongress-295499\#fn_636\#ixzz0o8U7RV6m.
} 
negociación comercial que pueda implicar en un momento u otro el movimiento de personas o bienes o el flujo de servicios o energía a través de las fronteras de los estados, tengan o no una finalidad comercial stricto sensu. Esta cláusula se ha interpretado ampliamente y en pro de la competencia federal, sobre todo a partir de los años treinta, sobre la base de la existencia de una "relación substancial" con el comercio, lo que acabó traduciéndose, de acuerdo con un importante sector doctrinal, en una progresiva invasión del ámbito de intervención de los estados por parte del legislador federal ${ }^{4}$.

Sin embargo, la aplicación de dicha cláusula al supuesto ambiental plantea algunas dudas sobre el alcance de la competencia federal desde la decisión del Tribunal Supremo en 1995 en el asunto United States v. Lopez ${ }^{5}$ en la que se cuestionaba la constitucionalidad de la Gun-Free School Act de 1990 por exceder la potestad del legislador federal. Aunque no puede afirmarse que ésta decisión implique un cambio sustancial de la jurisprudencia del Tribunal Supremo, hay que señalar la importancia que tiene en la determinación de la sede federal o estatal para legislar en materia de medio ambiente, al menos respecto a aquellas actividades que se caracterizan por la ausencia de una dimensión comercial que afecte a las relaciones inter-estatales ${ }^{6}$. La jurisprudencia ha seguido esta línea en varias ocasiones en que los operadores económicos han atacado normativas ambientales federales que regulaban actividades con alcance inter-estatal, pero que carecían de conexión comercial ${ }^{7}$.

\footnotetext{
${ }^{4}$ FABER, A., "The Constitution's Forgotten Cover Setter: An Essay on the New Federalism and the Original Understanding", en Michigan Law Review, núm. 94, 1995, p. 610; FEREJOHN, J.-WEINGAST, B.R. (Ed.), The New Federalism. Can the States be Trusted?, Hoover Institution Press-Stanford University, Stanford, 1997.

${ }^{5}$ Sentencia de la Corte Suprema de 26.4.1994, 514 US 549 (1995), en Conwell University Law School, Legal Information Institute, recuperado el 25 diciembre $2010 \mathrm{de}$ http://www.law.cornell.edu/supct/html/ 93-1260.ZO.html. Vid. al respecto, REY MARTÍNEZ,F., "United States v. Lopez y el nuevo federalismo americano", en Revista Española de Derecho Constitucional, núm. 51, 1997, p. 273; CALABRESI, S.G., "A Government of Limited and Enumerated Powers: In Defense of United status v. López", en Michigan Law Review, núm. 94, 1995, p. 752; GRAGLIA, L.A., "United States v. Lopez: Judicial Review Ander the Comerse Clause", en Texas Law Review, núm. 74, 1995, p. 720.
}

6 TARLOK, D., "The Influence of International Environmental Law on United States Pollution Control Law", en Vermont Law Review, núm. 21,, 1997, p. 759, p. 761; PERCIVAL, R., "Environmental Law in the Twenty-First Century", en Virginia Environmental Law Journal, núm. 25, 2007, p. 1, p. 10 y ss.

7 Por ejemplo, United States v. Olin Corp., 107 F.3d 1506 (11 th Cir., 1997); National Associations of Home Builders v. Babbit, 130 F.3d 1041 (D.C. Cir., 1997); Gibbs v. Babbit, 214 F.3d 483 (4th Cir. 2000); GDF Reality Inv., Ltd. V. Norton, 326 F.3d 622 (5 ${ }^{\text {th }}$ Cir. 2003); Solid Waste Agency of N. Cook County (SWANCC) v. US Army Corps of Engineers, 531 US 159 (2001); Nebraska v. EPA, 331 F.3d 995 (DC Cir. 2003), en FindLaw, recuperado el 25 diciembre 2010 de http://caselaw.lp.findlaw.com. 
Por otro lado, conforme al mismo artículo I.8 de la Constitución, el Congreso tiene el poder para adoptar todas aquellas medidas que "shall be necessary and proper for carrying into execution the foregoing powers, and all other powers vested by this Constitution in the government of the United States, or in any department or officer thereof." Esta cláusula de poderes implícitos ha permitido una interpretación amplia y extensiva de las competencias del Congreso americano, que cubre incluso aquellas materias que no han sido explícitamente enumeradas por la Constitución, y que ha justificado de nuevo la intervención federal en ámbitos como la aviación, las telecomunicaciones, los ferrocarriles, o el derecho de la competencia. En base a esta misma competencia, la acción federal en materia de lucha contra el cambio climático, y en particular, para el establecimiento de un sistema de comercio de emisiones de gases de efecto invernadero (en adelante GEI) se ha intensificado en los últimos años mediante diversas propuestas, hasta ahora todas ellas fallidas.

La primera de las propuestas a las que hay que hacer referencia es la presentada ante el Senado el 18 de octubre de 2007 por el senador independiente Joseph Lieberman con el co-patrocinio del senador republicano John Warner (America's Climate Security Act) ${ }^{8}$. Si bien el proyecto fue informado favorablemente por el Comité de Medio Ambiente y Obras Públicas del Senado el 5 de diciembre del mismo año, el rechazo de los senadores republicanos y la finalización del período de sesiones del Congreso antes de que el proyecto de ley pasara a la Cámara de los Representantes, hizo que éste decayera y no se reintrodujo en el nuevo período de sesiones. Su finalidad última era lograr para el año 2050 una reducción de las emisiones de GEI del 70\% respecto al año de referencia de 2005. La segunda de las propuestas vino de la mano de dos representantes demócratas, Henry Waxman y Edward Markey (American Clean Energy and Security Act) ${ }^{9}$, con el apoyo de un número muy importante de asociaciones y organizaciones ambientalistas ${ }^{10}$.

\footnotetext{
8 S. 2191: The Climate Security Act, en Govtrack.us, Text of S. 2191 [110th]: Lieberman-Warner Climate Security Act of 2007, recuperado el 25 diciembre 2010 de http://www.govtrack.us/congress/ billtext.xpd?bill=s110-2191.

${ }^{9}$ H.R. 2454: The American Clean Energy and Security Act, en Govtrack.us, Text of H.R. 2454: American Clean Energy and Security Act of 2009, recuperado el 25 diciembre 2010 de http://www.govtrack.us/ congress/billtext.xpd?bill=h111-2454.

10 Entre otras, Defenders of Wildlife, Alliance for Climate Protection, Environmental Defense Fund, National Wildlife Federation, The Nature Conservancy, Audubon Society, Natural Resources Defense Council y el Sierra Club.
} 
El proyecto se introdujo en la Cámara de Representantes el 15 de mayo de 2009 y fue aprobado por el Pleno el 26 de junio con 219 votos a favor y 212 votos en contra ${ }^{11}$. El 7 de julio de 2009 pasó al Senado, donde no obtuvo la mayoría necesaria para permitir que avanzara el procedimiento. El objetivo principal de esta propuesta era la reducción global de las emisiones de GEI en un 20\% en 2020 respecto de los niveles existentes en 2005, hasta lograr en 2050 una reducción del 83\% respecto de los niveles de 2005. La tercera de las propuestas surge también del Senado, y fue presentada el 30 de septiembre de 2009 por los senadores demócratas John Kerry y Barbara Boxer (Clean Energy Jobs and American Power Act) ${ }^{12}$. A pesar del rechazo de los republicanos, el proyecto de ley fue informado por el Comité de Medio Ambiente y Obras Públicas el 5 de noviembre de 2009, y se incorporó al orden del día del Senado el 2 de febrero de 2010. No obstante, desde entonces no se ha producido ningún avance y se puede considerar un nuevo proyecto abandonado. Los objetivos de reducción de GEI de esta propuesta eran más estrictos que los de las anteriores, por lo menos en su primera fase: una reducción para 2020 del $20 \%$ respecto de los niveles existentes en 2005, hasta alcanzar un $-83 \%$ en 2050 .

Entre las iniciativas vinculadas con la lucha contra el cambio climático destaca la encabezada más recientemente por los senadores Lieberman (independiente), Kerry (demócrata) y Graham (republicano), la American Power Act ${ }^{13}$, conocida inicialmente como la tripartisan bill por el triple apoyo con el que contaba. Sin embargo, el senador Graham retiró posteriormente su apoyo y el proyecto se presentó al Senado el 12 de

\footnotetext{
${ }^{11}$ En The Library of Congress, Bill Summary \& Status 111th Congress (2009 - 2010) H.R.2454 Major Congressional Actions, recuperado el 25 diciembre 2010 de http://thomas.loc.gov/cgi-bin/bdquery/ z?d111:HR02454:@@@.

12 S. 1733: The Clean Energy Jobs and American Power Act, en Govtrack.us, Text of S. 1733: Clean Energy Jobs and American Power Act, recuperado el 25 diciembre 2010 de http://www.govtrack.us/ congress/billtext.xpd?bill=s111-1733.

13 American Power Act, en John Kerry US Senator web, APA Bill Text, recuperado el 25 diciembre 2010 de http://kerry.senate.gov/americanpoweract/pdf/APAbill.pdf. Con posterioridad se han presentado otras dos propuestas ante el Senado, que ya han quedado fuera de nuestro studio. Se trata de la propuesta presentada el 9 de junio de 2010 por el senador Lugar (Practical Energy and Climate Plan, S. 3464, en Govtrack.us, S. 3464, Practical Energy and Climate Plan Act of 2010, recuperado el 25 diciembre 2010 de http://www.govtrack.us/congress/billtext.xpd?bill=s111-3464) y la propuesta presentada el 21 de septiembre de 2010 por los senadores Bingaman,Dorgan -demócratas-, Brownback y Collins republicanos- (Renewable Energy Promotion Act, S.3813, en Govtrack.us, S. 3813, Renewable Electricity Promotion Act of 2010, recuperado elm 25 diciembre 2010 de http://www.govtrack.us/congress/bill.xpd? bill $=\mathrm{s} 111-3813)$.
} 
mayo de 2010 sin el respaldo de los republicanos, lo que ha dificultado su adopción final en esta institución.

La propuesta es una muestra de cómo el legislador federal, en su compromiso de desarrollar un sistema de comercio de emisiones de GEI, ha priorizado las concesiones a la industria energética y petrolera frente al cumplimiento de las obligaciones internacionales. Así parecen indicarlo al menos el establecimiento de unas cuotas de emisión aparentemente menos restrictivas que las contempladas en iniciativas anteriores, la limitación del número de sectores afectados o los amplios regímenes de exención. En la misma línea, el proyecto de ley incentiva y promueve el recurso extensivo a la energía nuclear, la industria de producción y distribución de gas natural y la extracción de petróleo en plataformas off-shore mediante la concesión de nuevos derechos de perforación sobre yacimientos submarinos.

De entrada, los objetivos cuantificados de reducción son menos exigentes que los recogidos en sus antecesoras. El proyecto de ley aspira ahora a recortar las emisiones en el año 2020 en un 17\% respecto del año de referencia de 2005, en un 42\% en 2030 y en un $80 \%$ en 2050 . El primer periodo de compromiso deberá iniciarse, no obstante, un año más tarde que en las propuestas anteriores, en 2013, año en el que debería lograrse una reducción del 4’75\%. Para facilitar estos objetivos de reducción la propuesta recoge, como en los casos anteriores, la creación de un mercado de derechos de emisión “cap \& trade".

En esta línea, el proyecto de ley se inclina por el establecimiento de umbrales de emisión sólo en determinadas instalaciones de generación eléctrica y determinadas instalaciones industriales (las primeras a partir de 2013, y las segundas a partir del 2016). Con respecto a la asignación de los derechos de emisión, el proyecto de ley prevé la subasta de una cantidad significativa, el $25 \%$ de los derechos de emisión. El porcentaje de los derechos que podrán subastarse se incrementará anualmente hasta lograr el 80\% en 2030, y el 100\% de los derechos en 2035. El proyecto de ley establece un precio mínimo de subasta, que inicialmente fija en 12\$ (en valor de 2009) para 2013, incrementándose en un 3\% anual por encima de la inflación.

El proyecto de ley prevé asimismo una reserva de derechos de emisión que, como en los casos anteriores, se introducirían en el mercado si se produjeran fluctuaciones 
inesperadas del precio de la tn $\mathrm{CO} 2 \mathrm{e}$ (con una limitación del 15\% y un precio que se fija en $25 \$$ en 2013 y que se incrementa anualmente en un 5\% por encima de la inflación). Los artículos 2401 a 2416 del proyecto de ley encargan a la Commodity Futures and Trading Commission la función de velar por el buen funcionamiento y la integridad de este mercado de emisiones, y de ocuparse de la fijación de las cantidades máximas de emisión, el establecimiento de los criterios de elegibilidad para participar al mercado, los procedimientos de gestión, etc.

Para facilitar la futura vinculación del sistema de comercio de derechos de emisión de GEI con otros sistemas que operen a escala internacional, el proyecto de ley prevé la participación de los operadores económicos en sistemas foráneos, siempre que dichos programas se ejecuten por una autoridad nacional o supranacional y sean comparables con el sistema norteamericano por su carácter obligatorio y su rigor. La posibilidad de obtención de créditos mediante otros proyectos y programas se autorizaría asimismo hasta un total de 2 billones de t. anuales. De estos créditos, sólo un $25 \%$ del total podría proceder de proyectos o programas internacionales. Conforme al proyecto de ley, estos créditos se valorarían en una relación $1=1$ hasta 2018 , y en una relación de $1.25=1$ a partir de entonces. Finalmente, como novedad respecto a las propuestas anteriores, el proyecto de ley permite, a partir de 2025 y como una salvaguarda adicional, la imposición de una tasa específica sobre determinadas mercancías (el acero, el aluminio o el cemento) procedentes de terceros Estados en los que no se aplican medidas similares de reducción de emisiones.

Por otro lado, las propuestas mencionadas tienen en común que todas ellas pretenden limitar el margen de actuación de la Environmental Protection Agency norteamericana (en adelante EPA) puesto que existe un cierto temor en el Congreso de que la reglamentación de la EPA, mucho más restrictiva, comporte un aumento de los precios de la energía sin la contrapartida de medidas positivas (comercio de derechos de emisión, promoción de la innovación tecnológica, compensaciones a la industria emisora, etc., $)^{14}$.

\footnotetext{
14 Esta ha sido una amenaza latente desde el mismo momento en el que el Presidente Obama anunció el 18 de Noviembre de 2008 el objetivo de reducción de las emisiones de GEI para el 2020 a los niveles de 1990. En Change.com. The Office of the President Elect, President-elect Obama promises "new chapter" on climate change, 18.11.2008, recuperado el 25 diciembre $2010 \mathrm{de} \mathrm{http://change.gov/newsroom/entry/}$ president_elect_obama_promises_new_chapter_on_climate_change/.
} 
El ejecutivo federal justifica la intervención de la EPA en la Clean Air Act de $1990^{15}$ y especialmente en la decisión del Tribunal Supremo del 2 de abril de 2007 en el asunto Massachusetts v. $E P A^{16}$. Dicho asunto se originó con la demanda de un grupo de organizaciones privadas contra la EPA por haberse negado a adoptar normas de regulación de las emisiones de GEI procedentes de los vehículos de motor ${ }^{17}$. El Tribunal Supremo entendió que los GEI quedaban cubiertos por la definición de contaminantes atmosféricos que recoge la Clean Air Act y requirió a la EPA para que se pronunciara sobre si estas emisiones podían causar o contribuir a una contaminación atmosférica que implicara un daño para la salud pública o el bienestar. Este pronunciamiento por sí mismo ya justificaba la intervención de la EPA, y la autorizaba a regular la emisión de estos gases más allá de que finalmente el Congreso fuera capaz o no de adoptar legislación al respecto.

En base a esta decisión, la EPA adoptó una primera medida ("endangerment finding") el 17 de abril de $2009^{18}$, confirmada posteriormente el 7 de diciembre del mismo año ${ }^{19}$, en la que calificaba los GEI como una amenaza para la salud pública y anunciaba que los reglamentaría conforme a la Clean Air Act. Junto con la National Highway Traffic and Safety Administration, la EPA presentó una primera propuesta sobre la reducción de las emisiones de GEI procedentes de los vehículos a motor ligeros el 28 de septiembre de

\footnotetext{
15 USC Title 42, Chapter 85, en US EPA, Clean Air Act, recuperado el 25 diciembre 2010 de http:// www.epa.gov/air/caa/.

${ }^{16}$ Massachusetts et al. v. Environmental Protection Agency et al., No. 05-1120.en Findlaw, recuperado el 25 diciembre 2010 de http://caselaw.lp.findlaw.com/scripts/getcase.pl? court $=$ US\&vol $=000 \&$ invol $=05-1120$.

17 El artículo 202, a, 1 de la Clean Air Act exige que la EPA "shall by regulation prescribe (...) standards applicable to the emission of any air pollutant from any class ... of new motor vehicles".

18 EPA, 40 CFR Chapter 1, [EPA-HQ-OAR-2009-0171; FRL-8895-5], Proposed Endangerment and Cause or Contribute Findings for Greenhouse Gases Under Section 202(a) of the Clean Air Act, en Federal Register, Vol. 74, No. 78, Friday, April 24, 2009, Proposed Rule, recuperado el 25 diciembre 2010 de http://www.epa.gov/climatechange/endangerment/downloads/EPA-HQ-OAR-2009-0171-0001.pdf.

19 EPA, 40 CFR Chapter I, [EPA-HQ-OAR-2009-0171; FRL-9091-8], Endangerment and Cause or Contribute Findings for Greenhouse Gases Under Section 202(a) of the Clean Air Act, en Federal Register, Vol. 74, No. 239, Tuesday, December 15, 2009, Rules and Regulations, recuperado el 25 diciembre de http:/www.epa.gov/climatechange/endangerment/downloads/Federal_Register-EPA-HQOAR-2009-0171-Dec.15-09.pdf.
} 
200920. Esta propuesta, cuya versión final se hizo pública el 14 de abril de $2010^{21}$, constituye el primer programa de reducción de las emisiones de GEI adoptado en el marco de la Clean Air Act ${ }^{22}$.

Paralelamente, el 5 de octubre de 2009, el Presidente Obama firmó la Executive Order EO $13514^{23}$, que exigía a las Agencias federales el establecimiento de objetivos cuantificados de reducción y limitación de las emisiones de GEI para el año 2020 y disponía una serie de medidas de promoción de los programas de eficiencia energética y reducción del consumo y dependencia del petróleo, entre otros. Con este objetivo, la EPA adoptó finalmente el 13 de mayo 2010 una nueva norma por la cual se establecen limitaciones específicas para la emisión de GEI, aplicable a determinadas instalaciones industriales sujetas a la Clean Air Act y que quedan cubiertas por los programas específicos de control de las emisiones ${ }^{24}$. En una primera fase, entre enero y junio de 2011, la norma afecta sólo a las instalaciones existentes que actualmente ya están cubiertas por la exigencia de la obtención de permisos de emisión en el contexto de otros programas de reducción de emisiones, si las emisiones son superiores a 75.000 t.3 anuales (con algunas excepciones en que la cantidad se fija en 25.000). Durante la segunda fase, entre julio de 2011 y julio de 2013, la exigencia de la obtención del permiso se extiende a las nuevas instalaciones, exigiéndose el uso de mejores

20 EPA, 40 CFR Parts 86 and 600, Department of Transportation, National Highway Traffic Safety Administration, 49 CFR Parts 531, 533, 537, and 538, [EPA-HQ-OAR-2009-0472; FRL-8959-4; NHTSA-2009-0059], Proposed Rulemaking To Establish Light-Duty Vehicle Greenhouse Gas Emission Standards and Corporate Average Fuel Economy Standards, en Federal Register, Vol. 74, No. 186, Monday, September 28, 2009, Proposed Rules, recuperado el 25 diciembre 2010 de http:// edocket.access.gpo.gov/2009/pdf/E9-22516.pdf.

${ }^{21}$ EPA and NHTSA Finalize Historic National Program to Reduce Greenhouse Gases and Improve Fuel Economy for Cars and Trucks, en EPA-420-F-10-014, April 2010, recuperado el 25 diciembre 2010 de http://www.epa.gov/oms/climate/regulations/420f10014.htm.

22 El 1 de abril de 2010 la EPA ya anunció una nueva medida en la misma línea, esta vez destinada a regular las emisiones procedentes de los vehiculos pesados. Vid., Canada and the United States Announce Common Standards for regulating GHG Emissions from New Vehicles, en Environment Canada, recuperado el 25 diciembre 2010 de http://ec.gc.ca/default.asp?lang=En\&n=714D9AAE-1\&news=B1DDFE4D-5147-46F9BA97-BA1BDDC3B7A6. Vid., también el reglamento en Government of Canada, Vol. 144, No. 16 - April 17, 2010, recuperado el 25 diciembre $2010 \mathrm{de} \mathrm{http://www.gazette.gc.ca/rp-pr/p1/2010/2010-04-17/html/reg1-}$ eng.html.

23 En The White House, Office of the Press Secretary, recuperado el 25 diciembre 2010 de http:// www.whitehouse.gov/assets/documents/2009fedleader_eo_rel.pdf.

24 Fact Sheet -- Proposed Rule: Prevention of Significant Deterioration and Title V Greenhouse Gas Tailoring Rule, en US EPA, recuperado el 25 diciembre 2010 de http://www.epa.gov/NSR/ fs20090930action.html. 
tecnologías disponibles de control y las medidas de eficiencia energética para reducir al mínimo las emisiones de GEI.

\section{El inmovilismo del poder federal en Canadá}

En el caso de Canadá nos interesa referir igualmente la existencia de una competencia federal que justifique la adopción de las medidas para asegurar el cumplimiento del compromiso de reducción del 6\% de los niveles de emisión de GEI asumido en el PK. Con respecto a la protección del medio ambiente, no obstante, la Constitución canadiense no menciona ninguna competencia específica cuando hace la clasificación de las materias que quedan dentro de la competencia del poder federal o del poder provincial. En la medida, pues, en que esta materia no se inscribe en un título competencial único ${ }^{25}$ ni se atribuye a una u otra instancia, tanto el legislador federal como el provincial ${ }^{26}$ pueden considerarse competentes en base a los poderes que tienen en materias conexas, lo que ha dado lugar a un importante debate que continúa dividiendo a los especialistas.

La adopción de medidas de protección ambiental por parte del legislador federal puede justificarse en las competencias que la Constitución le atribuye en el artículo 91 en materia de intercambios comerciales, política fiscal, navegación, pesquerías, patentes de invenciones y descubrimientos, territorios y reservas indias, así como la tipificación de los delitos e imposición de sanciones penales. No obstante, el alcance de estas competencias se puede ver modificado por la interpretación jurisprudencial de la cláusula relativa a la paz, orden y buen gobierno ("peace, order and good government") que contiene el párrafo introductorio del artículo 91. La cuestión que se plantea entonces es la de determinar si dicha cláusula permite priorizar la intervención del Parlamento federal en materias que si bien afectan a competencias provinciales, superan los límites de la provincia, o si, al contrario, sólo puede atribuirse al legislador federal

\footnotetext{
25 Vid., BEAUDOIN, G.A, « La protection de l'environnement et ses implications en droit constitutionnel», en McGill Law Journal, núm. 23, 1977, p. 207.

${ }^{26}$ GIBSON,D. "Constitutional Jurisdiction over Environmental Management in Canada", en University of Toronto Law Journal, núm. 23, 1973, p. 54.
} 
esta competencia cuando se puede tratar separadamente, desvinculándose de las competencias de las provincias enumeradas en el artículo 92 de la Constitución ${ }^{27}$.

Este es en parte el objeto de la sentencia de 24 de marzo de 1988 en el asunto Crown Zellerbach Canada Ltd ${ }^{28}$, en la que el Tribunal Supremo consideró que la regulación de la contaminación marítima puede ser objeto de la competencia exclusiva del Parlamento federal por su incidencia supra provincial e internacional. En esta sentencia, el Tribunal Supremo entendió que para afirmar la competencia federal eran necesarios dos elementos: a) El ámbito de acción debía presentar un grado suficiente de singularidad, carácter diferenciado e indivisibilidad respecto a la acción provincial y b) El provincial inability test, esto es, debía valorarse la incapacidad de la provincia para la regulación de los aspectos intra-provinciales de la materia y sus consecuencias para los intereses extra-provinciales ${ }^{29}$. Ambos aspectos parecen estar presentes en la regulación de los GEI, lo que permitiría justificar la intervención federal concurrente sobre la base de la doctrina de la cláusula relativa a la paz, orden y buen gobierno, siempre que ésta se ciña a los "ascertainable and reasonable limits, in so far as its impact on provincial jurisdiction is concerned", tal y como indica la sentencia mencionada ${ }^{30}$. Las mismas consecuencias internacionales del fenómeno del cambio climático, la dimensión del compromiso asumido en el PK, el riesgo de deslocalización de las emisiones hacia sectores y espacios no regulados y la insuficiencia de una respuesta únicamente provincial apuntan a la misma solución ${ }^{31}$.

\footnotetext{
27 Sobre ambas posiciones Vid., PATENAUDE, M., "L'interprétation du partage des compétences à l'heure du libre-échange", en Revue de Droit de l'Université de Sherbrooke., núm. 21, 1990, p. 1.

${ }^{28}$ R. v. Crown Zellerbach Canada Ltd., [1988] 1 S.C.R. 401 en Biblioteca de la Université de Montréal, LexUM, recuperado el 25 diciembre 2010 de http://scc.lexum.umontreal.ca/en/ 1988/1988scr1-401/1988scr1-401.pdf.

29 Ibidem., par. 33, en Biblioteca de la Université de Montréal, LexUM, recuperado el 25 diciembre 2010 de http://scc.lexum.umontreal.ca/en/1988/1988scr1-401/1988scr1-401.pdf.

30 Ibidem. par.39. Vid. ROLFE, C., Turning Down the Heat: Emissions Trading and Canadian Implementation of the Kyoto Protocol, West Coast Environmental Law Research Foundation, Vancouver, 1998, p. 352 y BARTON, P., "Economic Instruments and the Kyoto Protocol: Can Parliament Implement Emissions Trading Without Provincial Co-operation?”, en Alberta Law Review, núm. 2, 2002, p. 417.

${ }^{31}$ WIENER, J.B., "Think Globally, Act Globally: The Limits of Local Climate Policies”, en University of Pennsylvania Law Review, núm. 155, 2007, p. 107; BACHELDER, A., "Using Credit Trading to Reduce Greenhouse Gas Emissions", en Environmental Law \& Practice, núm. 9, 2000, p. 281; WAKULAT, R., Canada's 2007 Climate Change Action Plan: Plan of Action or More Hot Air?, University of Toronto, 2008, p. 34, en http://www.cba.org/CBA/newsletters/pdf/10_08_env-essay.pdf.
} 
Esta misma cuestión fue de nuevo objeto de otra importante sentencia el 18 de septiembre de 1997, en el asunto R. V. Hydro-Québec ${ }^{32}$, en el cual el Tribunal Supremo desarrolló un segundo argumento. Así, entendió que la normativa federal aplicable a las sustancias tóxicas se justificaba en virtud de la competencia que la Constitución reconocía al legislativo federal en materia penal. Si bien la protección del medio ambiente constituye " $a$ diffuse subject that cuts across many different areas of constitutional responsibility, some federal, some provincial', el juez La Forest, en nombre de la mayoría, consideró que “(...) It is surely an "interest threatened” which Parliament can legitimately "safeguard", or to put it another way, pollution is an "evil" that Parliament can legitimately seek to suppress. (...) The criminal law must be able to keep pace with and protect our emerging values"33. La jurisprudencia en el asunto Hydro-Québec permite sostener que la protección ambiental puede ser también uno de los objetivos de las normas penales en el marco del mantenimiento de la paz, el orden, la seguridad, la salud o la moralidad públicas. Por lo tanto, cabe plantear si el gobierno federal puede justificar su intervención en el marco de la imposición de sanciones específicas para implementar la prohibición de emisiones de GEI por encima de determinados niveles, lo que por sí solo se puede considerar un atentado al medio ambiente que comporta un "mal público" 34. Cómo ha señalado HÉLÈNE TRUDEAU 35 , la respuesta debe ser positiva ya que, de la misma manera que ocurrió en su momento con las substancias que agotan la capa de ozono, los GEI pueden considerarse sustancias toxicas en el sentido de la la Canadian Environmental Protection Act de

32 R. v. HydroQuébec, [1997] 3 R.C.S. 213 en Biblioteca de la Université de Montréal, LexUM, recuperado el 25 diciembre 2010 de http://csc.lexum.umontreal.ca/en/ 1997/1997scr3-213/1997scr3-213.html. Vid., DEIMANN, S., "R. v. Hydro-Québec: Federal Environmental Regulation as Criminal Law”, en McGill Law Journal, núm. 43, 1998, p. 923.

${ }^{33}$ R. v. HydroQuébec, [1997] 3 R.C.S. 213, par. 123, 127 y 128

34 Vid. LECLAIR,J., «Aperçu des virtualités de la compétence fédérale en droit criminel dans le contexte de la protection de l'environnement», en Revue Générale de Droit, núm. 27, 1996, p. 137. Ver asimismo, respecto al riesgo de una extension excesiva de la intervención federal, BENIDICKSON, J., Environmental Law, $3^{\text {rd }}$ edition, Irwin Law, Toronto, 2009, p. 34; ROLFE,C., Turning Down the Heat: Emissions Trading and Canadian Implementation of the Kyoto Protocol, West Coast Environmental Law Research Foundation, Vancouver, 1998, p. 352; BARTON, P., "Economic Instruments and the Kyoto Protocol: Can Parliament Implement Emissions Trading Without Provincial Co-operation?", en Alberta Law Review, núm. 2, 2002, p. 417; CHALIFOUR,N., "Making Federalism Work for Climate Change: Canada's Division of Powers over Carbon Taxes", en National Journal of Constitutional Law, núm 22, 2008,p. 119.

35 TRUDEAU,H..- LALONDE, S., « La Mise en Oeuvre du Protocole de Kyoto au Canada: Concertation ou Coercition », en Revue générale de droit, núm. 34, 2004, p. 141, p. 178. 
199936. Sería difícil argumentar que el Parlamento federal no pudiera seguir el mismo criterio en la lucha contra el calentamiento global, dada la similitud entre los efectos de ambos fenómenos. Si esta respuesta es afirmativa, cabe entonces la posibilidad de recurrir a la competencia en materia penal para justificar la adopción de medidas federales, siempre que se confirmen los criterios de legitimidad del objetivo público que persiguen y de la inclusión de sanciones para garantizar el cumplimiento de la prohibición ${ }^{37}$.

Por otro lado, desde la perspectiva del desarrollo normativo, la acción reciente del gobierno canadiense se ha caracterizado por dos aspectos. De una parte, la administración federal entiende estas actuaciones en el marco de una política común norteamericana para limitar las emisiones de GEI, lo cual desde febrero de 2009 tiene lugar en el seno del proceso "Clean Energy Dialogue"38. Sin embargo, la iniciativa ha correspondido hasta ahora a Estados Unidos. Canadá ha justificado tradicionalmente su posición más renuente en la vinculación entre la economía canadiense y la estadounidense y en los problemas de competitividad que puede comportar una política de reducción de emisiones diferenciada. En la práctica esto ha revertido en una dependencia excesiva respecto de la política de lucha contra el cambio climático de la administración de los Estados Unidos, tanto a nivel interno como en el escenario internacional. De otra parte, la acción del gobierno federal ha sido errática e incoherente y se ha traducido en múltiples propuestas poco exitosas ${ }^{39}$. Entre estas acciones desataca la adopción el 2007 del plan Turning the Corner: An Action Plan to Reduce Greenhouse

\footnotetext{
36 S.C. 1999, c. 33, en Government of Canada, Minister of Justice, recuperado el 25 diciembre 2010 de http://laws.justice.gc.ca/PDF/Statute/C/C-15.31.pdf.

${ }^{37}$ TRUDEAU,H..- LALONDE, S., « La Mise en Oeuvre du Protocole de Kyoto au Canada: Concertation ou Coercition », Op.cit., p. 179.

38 En Government of Canada, Canada's Action on Climate Change, recuperado el 25 diciembre 2010 de http://climatechange.gc.ca/default.asp?lang=En\&n=BDE8CD02-1.

39 National Action Program on Climate Change, Ottawa, 1995; Action Plan 2000 on Climate Change, Ottawa, 2000; Climate Change Plan for Canada: Achieving our Commitments Together, Ottawa, 2002; Moving Forward on Climate Change: A Plan for Honouring our Kyoto Commitment, Ottawa, 2005; Turning the Corner: An Action Plan to Reduce Greenhouse Gases and Air Pollution, Ottawa, 2007. Sobre este proceso, Vid., SMITH,H.A., "Political parties and Canadian climate change policy", en International Journal, núm. 64, 2008-2009, p. 47; SIMPSON,J.- JACCARD,M.-RIVERS,N., Hot Air: Meeting Canada's Climate Change Challenge, McClelland \& Stewart Ltd., Toronto, 2007, p. 91.
} 
Gases and Air Pollution por el actual gobierno conservador ${ }^{40}$, en la línea que había impuesto hasta entonces la administración Bush en Estados Unidos. De hecho, el plan de 2007 no se presentó nunca desde la perspectiva del cumplimiento del compromiso de reducción de emisiones asumido por Canadá en el $\mathrm{PK}^{41}$, lo que ha llevado al gobierno federal, actualmente en minoría, a enfrentarse a varias iniciativas procedentes de los partidos de la oposición. Por ejemplo, el Act to ensure Canada meets its global climate change obligations under the Kyoto Protocol (Act 288) ${ }^{42}$ de julio de 2007, exigió que "Canada takes effective and timely action to meet its obligations under the Kyoto

\footnotetext{
${ }^{40}$ Turning the Corner: An Action Plan to Reduce Greenhouse Gases and Air Pollution, en Government of Canada, Environment Canada, recuperado el 25 de diciembre 2010 de http://www.ec.gc.ca/default.asp? lang=En\&n=4891B242-1. Como principales resultados se pueden señalar dos: El primero hace referencia al establecimiento de objetivos de reducción de la intensidad de las emisiones; así, a largo plazo se adoptó el objetivo de reducir al año 2020 los GEI un $20 \%$ respecto del año de referencia de 2006, y lograr entre un 60 y un $70 \%$ de reducción en el año 2050 (Government of Canada, Bill C-30, The Clean Air Act, 2006, recuperado el 25 de diciembre 2010 de http://www.parl.gc.ca/common/bills_ls.asp? lang $=E \& l s=c 30 \&$ source $=$ library_prb\&Parl $=39 \& S e s=1)$; a corto plazo, el objetivo era reducir las emisiones de los grandes emisores en un 6\% cada año desde 2007 hasta 2010 y en un $2 \%$ por año cada año subsiguiente. El Plan también ha comportado la adopción de un nuevo marco reglamentario para las emisiones atmosféricas el 25 de Abril de 2008 (Cadre Reglementaire sur las émissions atmosfériques, en Government of Canada, Environment Canada, recuperado el 25 de diciembre 2010 de http:// www.ec.gc.ca/doc/media/m_124/toc fra.htm), si bien éste establecía únicamente objetivos de reducción de la intensidad de las emisiones y no objetivos absolutos de reducción. En la misma línea debe señalarse el anuncio hecho por el gobierno canadiense en abril y mayo de 2010 respecto de la adopción de reglamentación relativa a la reducción de las emisiones de GEI procedentes de los vehículos ligeros (Notice of intent to develop regulations limiting carbon dioxide emisiones from new cars and light-duty truck, Canada Gazette, Part I, en Government of Canada, Environment Canada, recuperado el 25 diciembre 2010 de www.ec.gc.ca/ceparegistry/notices/noticedetail.cfm?intNotice=500. El texto de la propuesta Passenger automobile and light truck greenhouse gas emission Regulations se puede encontrar también en Government of Canada, Canada Gazette, recuperado el 25 diciembre 2010 de http:// www.gazette.gc.ca/rp-pr/p1/2010/2010-04-17/html/reg1-eng.html) y la relativa a los vehículos pesados (Canada Announces Continental Approach to Reduce GHG Emissions from Heavy-Duty Vehicles, en Government of Canada, Environment Canada, recuperado el 25 diciembre 2010 de http://www.ec.gc.ca/ default.asp?lang=En\&n=714D9AAE-1\&news=2D7A8979-B4F4-4A06-87E0-C76237F5E803 ).
}

41 Turning the Corner: An Action Plan to Reduce Greenhouse Gases and Air Pollution, en Government of Canada, recuperado el 25 diciembre 2010 de http://www.ec.gc.ca/doc/virage-corner/2008-03/pdf/ COM-541_Framework.pdf, p. 8.

42 S.C. 2007, c. 30, en GLIN, An Act to ensure Canada meets its global climate change obligations under the Kyoto Protocol (Kyoto Protocol Implementation Act), recuperado el 25 diciembre 2010 de http:// www.glin.gov/view.action?glinID=207831. 
Protocol and help address the problem of global climate change"43. Otro ejemplo de la acción de la oposición en la misma línea es la Act to Ensure Canada Assumes its Responsibilities in Preventing Dangerous Climate Change ${ }^{44}$, adoptada por la Cámara de los Comunes el 5 de mayo de 2010 por 149 votos a favor y 136 votos en contra, lo que muestra la fuerte división política en torno a esta cuestión ${ }^{45}$.

\section{EL DINAMISMO DE LAS ENTIDADES SUB-NACIONALES EN MATERIA DE CAMBIO CLIMÁTICO EN NORTEAMÉRICA}

\section{Una intervención de los estados basada en la cooperación entre administraciones en Estados Unidos}

En materia de protección del medio ambiente, la concurrencia de la competencia entre los estados y el legislador federal ha experimentado un cierto retroceso en los últimos $a_{n ̃ o s}{ }^{46}$. La intervención federal ha sido amplia, incluso en supuestos en los que el problema ambiental al que responde la acción federal carece de una dimensión

\footnotetext{
43 La inacción del gobierno federal motivó el planteamiento ante los tribunales federales de un procedimiento judicial por el inclumplimiento de la Kyoto Protocol Implementation Act. No obstante, la Federal Court Trial Division decidió el 20 de octubre de 2008 (Federal Court, Friends of the Earth v. Canada, 2008 en FC 1183, [2009] 3 F.C.R. 201, recuperado el 25 diciembre 2010 de http:// reports.fja.gc.ca/eng/2008/2008fc1183/2008fc1183.pdf) que la mencionada ley se limitaba a crear un sistema público de rendimiento de cuentas y lo que en definitiva planteaba eran cuestiones políticas que debían resolverse en el foro político del Parlamento y no ante los tribunales. El 28 de octubre de 2009 la Federal Court of Appeal (Federal Court of Appeal, 15-10-2009, 2009 FCA 297, Friends of the Earth $v$. Canada, en Federal Court of Appel, Canada, recuperado el 25 diciembre 2010 de http://decisions.fcacaf.gc.ca/en/2009/2009fca297/2009fca297.pdf) confirmó esta decisión y rechazó el recurso de apelación de Friends of the Earth. El 15 de diciembre de este año, la asociación interpuso un nuevo recurso de apelación ante el Tribunal Supremo de Canadá (en Supreme Court of Canada, Case Information Summary 33469, Friends of the Earth v. Canada, recuperado el 25 diciembre 2010 de
}

http://www.scc-csc.gc.ca/case-dossier/cms-sgd/dock-regi-eng.aspx?cas=33469) que fue rechazado el 25 de Marzo 2010 (Supreme Court of Canada, Judgments in Leave Applications, recuperado el 25 diciembre $2010 \mathrm{de} \mathrm{http://scc.lexum.umontreal.ca/en/news} \mathrm{release/2010/10-03-25.3a/10-03-25.3a.html).}$

${ }^{44}$ House of Commons, $2^{\text {nd }}$ session, 40 ${ }^{\text {th }}$ Parliament, Bill C-311, An Act to ensure Canada assumes its responsibilities in preventing dangerous climate change, en Parliament of Canada, recuperado el 25 diciembre 2010 de

http://www2.parl.gc.ca/HousePublications/Publication.aspx?DocId=3662654\&Language=e\&Mode=1

45 En Parliament of Canada, recuperado el 25 diciembre 2010 de

http://www2.parl.gc.ca/HousePublications/Publication.aspx?DocId=4501250\&Language=e\&Mode=1

46 SMITH, J.-VOGEL, J.-CRUCE, T., SEIDEL, S.- HOLSINGER, Adapting to Climate Change. A Call for Federal Leadership, Pew Center on Global Climate Change- The Rockefeller Foundation, April 2010, en http://www.pewclimate.org/docUploads/adaptation-federal-leadership.pdf, p. 6 y ss. 
interestatal ${ }^{47}$; a su vez, es cada vez más frecuente que la legislación federal ambiental explicite su prevalencia sobre la legislación estatal, lo que se ha agravado por la interpretación extensiva que el Tribunal Supremo ha ido haciendo del principio de la preemption $^{48}$. Ello no obstante, en materia de medio ambiente y en particular respecto a la adopción de medidas de lucha contra el cambio climático, la acción estatal ha sido especialmente relevante.

El debate es antiguo y gira alrededor de la limitación de la intervención de las autoridades federales a un nivel que garantice el respeto y el mantenimiento de la diversidad de políticas públicas estatales. Desde un punto de vista tradicional se ha querido priorizar la autonomía, el enfoque regulador múltiple y las interacciones entre los poderes federal y estatal ${ }^{49}$, de modo que el ejercicio de la competencia normativa ha recaído normalmente en aquel nivel - estatal o federal- en el cual el conflicto ambiental, por su dimensión, podía ser mejor regulado. Así, la reglamentación ambiental tiende a ser federal cuando el proceso decisorio estatal o local no internaliza suficientemente los costes y los beneficios de la acción normativa o de la carencia de acción, un elemento que aparece con frecuencia respecto a algunas cuestiones ambientales con alcance interestatal ${ }^{50}$.

No obstante, se ha querido evitar una generalización excesiva y se ha insistido en la necesidad del examen caso a caso, tomando en consideración, entre otros elementos, la naturaleza y el alcance específico del daño ambiental, la complejidad técnica de la cuestión ambiental que se quiere resolver, el establecimiento o no de umbrales de

${ }^{47}$ Vid. CASTELLA ANDREU, J.M, "Tribunal Supremo y cambios en el federalismo de Estados Unidos", Op. Cit. p. 510 y ss.

48 Vid. ADELMAN,D.E.-ENGEL,K.H., "Adaptive Federalism: The Case Against Reallocating Environmental Regulatory Authority", en Arizona Legal Studies, Discussion Paper No. 07-23, September 2007, nota 3 y p. 40 y ss., en The University of Arizona, recuperado el 25 diciembre 2010 de http:// www.law.arizona.edu/faculty/FacultyPubs/Documents/Adelman/ALS07-23.pdf; CASTELLA ANDREU, J.M, "Tribunal Supremo y cambios en el federalismo de Estados Unidos", Op. Cit. p. 503 y ss.; SCHRAMM, D.P., "Federal Midwife: Assisting the States in the Birth of a National Greenhouse Gas Cap-and-Trade Program", en Tulane Environmental Law Journal, núm. 22, 2008, p. 61.

49 RABE, B., North American Federalism and Climate Change Policy: American State and Canadian Provincial Policy Development, en Widener Law Journal, núm.14, 2004, p. 121.

${ }^{50}$ STEWART, R.B., "Pyramids of Sacrifice? Problems of Federalism in Mandating State Implementations of National Environmental policy”, en Yale Law Journal, núm.6, 1977, p. 1196, en p. 1215 y ss. 
tolerancia, y la influencia de determinados grupos de interés ${ }^{51}$. También se ha señalado la vertiente dinámica del ejercicio de las competencias ambientales en el sistema federal americano. Así, a pesar del carácter multidisciplinar del fenómeno ambiental, se rechaza identificar una solución óptima que se pueda hacer extensiva de forma general a todos los supuestos. Esto implica adaptar el ejercicio de las competencias ambientales tanto a la variabilidad y a la diversidad estatal y local como a la misma heterogeneidad que presentan los problemas ambientales, promoviendo su desarrollo paralelo en los múltiples niveles de gobierno y generando varias opciones de acción pública ${ }^{52}$.

En particular, el carácter internacional de los retos ambientales que presenta el fenómeno del cambio climático parecería limitar la posibilidad de intervención de las autoridades estatales en Estados Unidos. Sin embargo, más allá del debate teórico, en la práctica han sido finalmente éstos y no el legislador federal quienes han adoptado la mayoría de las medidas de lucha contra el calentamiento global ${ }^{53}$. De una parte, la Constitución les permite la adopción de políticas y medidas conforme a sus particulares prioridades, también en materia de protección del medio ambiente y muy especialmente respecto a la lucha contra el cambio climático ${ }^{54}$. De la otra, la ausencia de programas federales a este respecto ha maximizado la intervención de los estados. Adalid en este

${ }^{51}$ ESTY, D., “Toward Optimal Environmental Governance", en New York University Law Review, núm. 74,1999 , p. 1495 , en p. 1554 y ss.

52 ADELMAN, D.E.-ENGEL, K.H., "Adaptive Federalism: The Case Against Reallocating Environmental Regulatory Authority", Op.Cit., en nota 3 y p.18 y ss., y p. 40 y ss.

53 Una recopilación de las medidas adoptadas por los cincuenta estados norteamericanos puede encontrarse en GERRARD, M.D. (ed.) Global Climate Change and US Law, American Bar Association, 2007, recuperado el 25 diciembre 2010 de http://www.abanet.org/abapubs/globalclimate/docs/ 50States_Survey_ThruNov09final.pdf. Algunas de estas iniciativas pueden encontrase asimismo en Pew Center on Global Climate Change, recuperado el 25 diciembre 2010 de http://www.pewclimate.org/ what_s_being_done/in_the_states/regional_initiatives.cfm. Vid. también KOSLOFF,L.-TREXLER,M.NELSOAN,H., "Facing Climate Change: Opportunities and Tools for State", en Widener Law Journal, 2004, p. 173; DERNBACH, J.C.- MCKINSTRY, R.B.- PETERSON, T.D., Making the States Full Partners in a National Climate Change Effort: A Necessary Element for Sustainable Economic Development, Paper presented at 2nd UNITAR-Yale Conference on Environmental Governance and Democracy, 17-19 September, New Haven, USA, en http://conference.unitar.org/yale/sites/ conference.unitar.org.yale/files/Paper_Dernbach.pdf, recuperado el 25 de diciembre 2010.

54 Esta posibilidad permite incluso la adopción de estándares de protección más restrictivos que los existentes a nivel federal, tal y como sucede en California en virtud de la cláusula de exención de la Clean Air Act de 1990 respecto a la regulación de la contaminación atmosférica. 
proceso ha sido sin duda el estado de California ${ }^{55}$, pero más de treinta estados han completado la elaboración de planes de acción contra el cambio climático, de los que veintidós contienen objetivos cuantificados de reducción de las emisiones de GEI ${ }^{56}$. Además, en cuarenta y dos estados se han establecido registros de las emisiones de GEI. Asimismo, mientras que a nivel federal se ha intentado avanzar sin mucha fortuna en la adopción de instrumentos legislativos para establecer un sistema de mercado de emisiones de GEI, por parte de varios de los estados norteamericanos han surgido últimamente varias iniciativas de alcance interestatal, a las que se han añadido también algunas de las provincias canadienses más activas en la lucha contra el cambio climático.

\section{Una intervención de las provincias basada en la exclusividad de la competencia en Canadá}

En Canadá la adopción de medidas en materia de protección ambiental por parte de las provincias se fundamenta en las competencias enunciadas en el artículo 92 de la Constitución, en particular en cuatro ámbitos $\operatorname{concretos}^{57}$ : la gestión del suelo público y de los recursos forestales provinciales, las instituciones municipales, el régimen jurídico de la propiedad y los derechos civiles, y la competencia residual que tienen las provincias sobre todas las materias de naturaleza puramente local o privada. Además, el

55 ROLAND-HOLST, D., Energy Efficiency, Innovation, and Job Creation in California; University of California, 2008, en Research Papers on Energy, Resources, and Economic Sustainability, Center for Energy, Resources, and Economic Sustainability (CERES), recuperado el 25 diciembre 2010 de http:// are.berkeley.edu/ dwrh/CERES Web/Docs/UCB\%20Energy\%20Innovation\%20and\%20Job\%20Creation \%2010-20-08.pdf.; DERNBACH, J. "Moving the Climate Debate from Models to Proposed Legislation: Lessons from State Experience", en Environmental Law Report, núm. 30, 2000, p. 10.933. En particular California ha mostrado siempre una posición bastante avanzada en relación al resto del pais. Ya en septiembre de 2000 adoptó la Ley 1771 (California Climate Action Registry), con el objetivo de stablecer objetivos cuantificados de emisión de GEI de carácter voluntario. Desde entonces se han adoptado varias leyes dirigidas a regular las emisiones de GEI (sobre las emisiones procedentes de los vehículos, sobre el secuestro de CO2, etc.) En particular, en septiembre de 2007 se adoptó la Ley 32 (California Global Warming Solutions Act) por la que se establece un programa de reducción de las emisiones de GEI en los que se combinan los instrumentos regulatorios con los mecanismos de mercado y en la que se fija el objetivo de lograr los niveles de emisión de 1990 en el año 2020.

56 Vid. la web del Pew Center on Global Climate Change, recuperada el 25 diciembre 2010 de http:// www.pewclimate.org; Vid. también Center for Climate Strategies-John Hopkins University, Impacts of Comprehensive Climate and Energy Policy Options on the U.S. Economy. Center for Climate Strategies, recuperado el 25 diciembre 2010 de http://www.climatestrategies.us/ewebeditpro/items/O25F23386.PDF.

57 Vid. TRUDEAU,H., "Le fédéralisme canadien et la protection de l'environnement”, en Souveraineté et intégration, Actes du Colloque conjoint des Facultés de Droit de l'Université de Poitiers et de l’Université de Montréal, Université de Montréal, Ed. Thémis, 1993, p. 229, en p. 235. 
artículo 92A de la Constitución otorga un poder adicional a las provincias, sin perjuicio de lo que pueda corresponder al Parlamento federal, al atribuirles la competencia legislativa en materia de prospección, explotación, conservación y gestión de los recursos naturales no renovables que se encuentran en su territorio.

La intervención de las provincias se produce también en relación con la ejecución de los tratados internacionales, y en el supuesto que ahora nos ocupa, del $\mathrm{PK}^{58}$. Por tanto, debe determinarse cuál es el alcance de la intervención de las asambleas legislativas de las provincias $^{59}$. El principio de base parece muy asentado desde la decisión en el asunto Labour Conventions de 28 de enero de 1937, en la que se hace depender la ejecución de las obligaciones internacionales de carácter convencional del reparto interno de las competencias legislativas impuesto por la Constitución ${ }^{60}$, una jurisprudencia que ha sido matizada por otras sentencias posteriores ${ }^{61}$. En definitiva, en Canadá el Parlamento federal no puede legislar para proceder a la aplicación de disposiciones de un tratado internacional que afecta a materias objeto de la competencia provincial. Esto supone, por lo menos en parte, un condicionamiento importante al ejercicio efectivo de las

58 DE MESTRAL, A.-FOX-DECENT,E., “ Rethinking the Relationship Between International and Domestic Law", en McGill Law Journal, núm. 53, 2008, p. 573, en par.20. La jurisprudencia de los tribunales canadienses ha sostenido tradicionalmente una perspectiva rigurosamente dualista. Vid., Ahani v. Canada (A.G.) (2002), 58 O.R (3rd 107, 208 D.L.R. (4th) 66 (C.A.); Baked v. Canada (Minister of Citizenship and Immigration); (1999) 2 S.C.R. 817, 174 D.L.R. (4th) 193; Bouzari v. Iran (2002) O.T.C. 297, 114, A.C.W.S. (3d) 57 (Ont.Sup.Ct), aff'd 71 O.R. (3d) 675, 243 D.L.R. (4 $\left.{ }^{\text {th }}\right) 406$ (C.A.); Canadian Foundation for Children, Youth and the Law v. Canada (A.G.) 2004 SCC 4, (2004) 1 S.C.R. 76, 234 D.L.R. (4 $\left.{ }^{\text {th }}\right)$ 257; National Corn Growers Assn. v. Canada (Import tribunal), (1990) 2 S.C.R. 1324, 74 D.L.R. (4 $\left.{ }^{\text {th }}\right)$ 449; Schreiber v. Canada (A.G.) 2002 SCC 62, (2002) 3 S.C.R. 269, 216 D.L.R. (4 $\left.4^{\text {th }}\right) 513$.

${ }^{59}$ Vid. BEAULAC, S., « National Application of International Law : The Statutory Interpretation Perspective », en Canadian Yearbook of International Law, núm. 41, 2004, p. 225; VAN ERT, G., "Using Treaties in Canadian Courts", en Canadian Yearbook of International Law, núm. 38, 2000, p. 3., en p. 28; DE MESTRAL, A.-FOX-DECENT,E., «Rethinking the Relationship Between International and Domestic Law», Op.Cit. par.58, 132, 133, 134, 158.

${ }^{60}$ Attorney-General of Canada v. Attorney-General of Ontario (Labour Conventions), [1937] A.C. 326

${ }^{61}$ MacDonald et al. v. Vapor Canada Ltd., [1977] 2 S.C.R. 134, p. 171 en Biblioteca de la Université de Montréal, LexUM, recuperado el 25 diciembre 2010 de http://scc.lexum.umontreal.ca/en/ 1976/1977scr2-134/1977scr2-134.html; Reference re Newfoundland Continental Shelf, [1984] 1 S.C.R. 86, Date: 1984-03-08, en Biblioteca de la Université de Montréal, LexUM, recuperado el 25 diciembre 2010 de http://scc.lexum.umontreal.ca/en/1984/1984scr1-86/1984scr1-86.html. 
competencias del Parlamento federal, que se ha manifestado de manera particularmente cruda en el caso del $\mathrm{PK}^{62}$.

Sin embargo, a pesar de disponer de un amplio margen de actuación, las provincias canadienses no han actuado de manera unánime. La concertación entre ellas ha sido difícil y de hecho a menudo han manifestado posiciones divergentes, en función de la percepción del impacto que sobre la competitividad y el crecimiento económico pueda tener la aplicación del PK. Este fenómeno responde también a las grandes disparidades existentes tradicionalmente en Canadá en materia de emisiones de GEI ${ }^{63}$. Desde esta perspectiva, parte del debate se ha centrado en la intensidad y el incremento de las emisiones de GEI en las distintas provincias (las emisiones del sector industrial son especialmente intensas, pero de crecimiento relativamente bajo, mientras que, por el contrario, las emisiones en el sector del petróleo, muy subvencionado, son de gran intensidad y de fuerte crecimiento), un debate que se ha centrado en las políticas respectivas de las provincias de Québec y Alberta. Esta valoración diferente se traduce también en el establecimiento de objetivos cuantificados de reducción de las emisiones de GEI que resultan muy diversos. Así, por ejemplo, el objetivo del gobierno de la provincia de Alberta, conforme a la Climate Change and Emisiones Management Act ${ }^{64}$, es el de lograr en 2020 una reducción del 50\% en la intensidad de las emisiones de GEI respecto de los niveles de 1990, mientras que, por el contrario, el gobierno provincial de la Columbia Británica persigue lograr en 2020 una reducción del 33\% respecto de los niveles de 2007 , lo que representa un $-10 \%$ respecto de los niveles de 1990; aquí sin

62 Vid. COHEN,M., "Canada and the International Legal Order: An Inside Perspective" , en MACDONALD,R.St.J.-MORRIS,G.L.-JOHNSTON,D.M. (ed.), Canadian Perspectives on International Law and Organization, University of Toronto Press, Toronto, 1974, p.3, en p. 16. En el mismo sentido crítico, HOWSE,R., "The Labour Convention Doctrine in an Era of Global Interdependence: Rethinking the Constitutional Dimensions of Canada's External Economic Relations", en Canadian Business Law Journal, núml. 16, 1990, p.160, en p.163 y ss; MORRIS, G.L., "Canadian Federalism and International Law”, en MACDONALD,R.St.J.-MORRIS,G.L.-JOHNSTON,D.M., Canadian Perspectives on International Law and Organization, Op. Cit., p. 55, en p. 58y ss.

${ }^{63} \mathrm{El}$ año 2008, por ejemplo, Alberta, con una fuerte industria petrolera y gasística, producía el 33,4\% del total de las emisiones del país y emitía cinco veces más GEI por habitante que Québec, que tiene como principal fuente de energía la hidroeléctrica, y donde las emisiones no superan el 13\% del total de Canadá. Vid. Environment Canada, Canada's 2008 Greenhouse Gas Inventory. A Summary of Trends: 1990-2008, en Government of Canada, Environment Canada, recuperado el 25 diciembre 2010 de http:// www.ec.gc.ca/ges-ghg/default.asp?lang=En\&n=0590640B-1.

64 Government of Alberta, Laws Online, Statutes of Alberta 2003, Chapter C-16.7, recuperado el 25 de diciembre $\quad 2010 \quad$ de $\quad$ ht t : / / w w w . alberta.ca/574.cfm? page $=$ C16P7.cfm\&leg_type $=$ Acts \&isbncln $=9780779740956$. 
embargo, a diferencia de lo que sucede en Alberta, el objetivo de reducción se expresa en términos absolutos. Ahora bien, este objetivo cuantificado es mucho más laxo que el que se fija en las provincias de Ontario, donde se quiere llegar a una reducción del 6\% de las emisiones en 2014, un $15 \%$ en 2020 y un $80 \%$ en 2050 en relación a los niveles de 1990, o de Québec, donde se espera una reducción del 6\% para 2012, respecto de los niveles de 1990.

\section{La acción de las entidades sub-nacionales y la articulación de redes transnacionales en Norteamérica}

La intervención de las entidades sub-nacionales y la descentralización con respecto a los mecanismos reguladores del comercio de emisiones de GEI son ya características de las medidas de lucha contra el cambio climático en Norteamérica. Esta perspectiva ha facilitado la creación y el rápido desarrollo, en este ámbito, de mecanismos de coordinación de las entidades sub-nacionales en forma de redes transnacionales ${ }^{65}$ que han adquirido un papel cada vez más relevante en el derecho internacional ${ }^{66}$. En el contexto norteamericano, en la materia que ahora nos ocupa, estas redes están sirviendo realmente para el establecimiento de estándares comunes más allá de la acción del poder

\footnotetext{
${ }^{65}$ Como indica RAUSTALIA, "They are "transgovernmental" because they involve specialized domestic officials directly interacting with each other, often with minimal supervision by foreign ministries. They are "networks" because this cooperation is based on loosely-structured, peer-to-peer ties developed through frequent interaction rather than formal negotiation. Thus defined, the phrase "transgovernmental networks" captures a strikingly wide array of contemporary cooperation", RAUSTIALA, K., "The Architecture of International Cooperation: Transgovernmental Networks and the Future of International Law", en Virginia Journal of International Law, núm. 43, 2002, p. 1, en p. 5. La metodología del trabajo en red a nivel transnacional no resulta tampoco extraña a la UE, que especialmente en materia de protección del medio ambiente laha promovido desde 2001 en el marco del Método Abierto de Cooperación. Vid. $\operatorname{COM(2005)} 706$ final. Vid. también DEHOUSSE,R., The Open Method of Coordination: A New Policy Paradigm?, Paper presented at the First Pan-European Conference on European Union Politics "The Politics of European Integration: Academic Acquis and Future Challenges," Bordeaux, septiembre de 2002, recuperado el 25 de diciembre 2010 de http:// eucenter.wisc.edu/OMC/Papers/Dehousse.pdf; RADAELLI,C.M., The Open Method of Coordination: A new governance architecture for the European Union?, Rapport nr. 1, Swedish Institute for European Policy Studies, 2003, recuperado el 25 de diciembre 2010 de http://eucenter.wisc.edu/OMC/Papers/ radaelli.pdf; VON HOMEYER,I.-KLASING,A-KREMER,A., Exploring the EU: Open Method of Coordination, Paper for the Workshop 'The Open Method of Co-ordination - Risks and Chances for European Environmental Policy', marzo 2004, Ecologic - Institute for international and European Environmental Policy, recuperado el 25 dediciembre 2010 de http://eucenter.wisc.edu/OMC/Papers/ Other/environment.pdf.
}

${ }^{66}$ RAUSTIALA, K., "The Architecture of International Cooperation: Transgovernmental Networks and the Future of International Law", Op.Cit.. en p. 23 y ss. y p. 76 y ss.; AHDIEH, R.,B., "Asuntos exteriores, derecho internacional y el nuevo federalismo: Lecciones derivadas de la coordinación", en Teoría y realidad constitucional, núm. 24, 2009, p. 109, en p. 111-112, 143 y ss. 
federal y han favorecido la implementación de tratados internacionales como el PK. En su vertiente internacional, además, no se puede ignorar el potencial que tienen para promover la convergencia de determinadas políticas ${ }^{67}$. Seguidamente se presentan brevemente las tres redes en las que participan actualmente estados y provincias de Estados Unidos y Canadá.

\subsection{Regional Greenhouse Gas Initiative 68}

La Regional Greenhouse Gas Initiative (en adelante RGGI) supone el establecimiento del primer programa de "cap \& trade" para las emisiones de $\mathrm{CO} 2$ de carácter obligatorio en Estados Unidos. Las primeras propuestas para la creación de esta red surgen en el año 2003, más o menos de manera paralela a la adopción en la UE de la Directiva 2003/87/CE, por la que se establece un régimen para el comercio de derechos de emisión de gases de efecto invernadero en la Comunidad ${ }^{69}$, pero no cristalizaron hasta diciembre de 2005 mediante un Memorandum of Understanding firmado por los gobernadores de siete estados de los Estados Unidos: Connecticut, Delaware, Maine, Nuevo Hampshire, Nueva Jersey, Nueva York, Vermont, Massachusetts, Rhode Island y Maryland ${ }^{70}$. Por parte canadiense se añadieron como miembros las provincias de Isla del Príncipe Eduardo, Terranova y Labrador. De otro lado, tienen el estatuto de observador Pennsylvania y el Distrito de Columbia en los Estados Unidos, y Québec, Nueva Escocia, New Brunswick y Ontario en Canadá.

La RGGI funciona mediante el establecimiento de un límite máximo de toneladas de $\mathrm{CO} 2$ y la distribución de los derechos de emisión entre las instalaciones de generación de energía, responsables aproximadamente del 95\% del total de las emisiones generadas en la región. El programa no es excesivamente ambicioso: limita las emisiones a los niveles de 2009, y se va reduciendo progresivamente hasta lograr un $-10 \%$ en 2018 . Con respecto a la forma de asignación de los derechos de emisiones, el programa difiere

${ }^{67}$ RAUSTIALA, K., "The Architecture of International Cooperation: Transgovernmental Networks and the Future of International Law", Op.Cit, en p. 49 y ss. y p. 89 y ss.

${ }^{68}$ Regional Greenhouse Gas Initiative, recuperado el 25 de diciembre 2010 de http://www.rggi.org/home.

${ }^{69}$ DOUE L 275, de 25.10.2003.

70 Regional Greenhouse Gas Initiative. Memorandum of Understanding, recuperado el 25 diciembre 2010 de http://rggi.org/docs/mou_12_20_05.pdf. 
bastante de las propuestas federales que actualmente se discuten en Estados Unidos y en Canadá, y directamente se pronuncia por la subasta del $100 \%$ de los derechos de emisión (con unos precios, no obstante, muy bajos: de los 3,07\$ en septiembre de 2008, han disminuido hasta los 2,05\$ en diciembre de 2009).

Otro de los elementos importantes en este sistema es el uso de los créditos que pueden obtenerse mediante la realización de proyectos de reducción o participando en programas similares. Así, las centrales eléctricas en los estados estadounidenses y las provincias canadienses miembros de la asociación pueden cumplir sus obligaciones en materia de reducción de las emisiones mediante la adquisición de un máximo del 3,3\% de las emisiones que les corresponden. Estos créditos se limitan a cinco categorías de proyectos: captura y destrucción de metano, reducción de las emisiones de SF6 en el sector de la energía eléctrica, secuestro de CO2 mediante la forestación, reducción de las emisiones de $\mathrm{CO} 2$ procedentes de la producción del gas natural, petróleo o gas propano de uso final y reducción de las emisiones de metano en las actividades agrícolas. También se permite la obtención de créditos procedentes de programas internacionales de comercio de emisiones o de proyectos que se lleven a cabo en otros territorios de Estados Unidos o Canadá que no son miembros de la asociación, siempre y cuando estos créditos se obtengan con "actions that are real, surplus, verifiable, permanent and enforceable"71.

A pesar de que todavía es demasiado pronto para valorar el éxito de la iniciativa y ha transcurrido poco más de un año desde su entrada en funcionamiento, por lo menos ha permitido iniciar un camino interesante de cooperación interestatal, que ha empezado a dar algunos resultados. Un ejemplo lo constituye el establecimiento de la RGGI CO2 Allowance Tracking System (RGGI COATS) ${ }^{72}$, la plataforma que registra y hace el seguimiento de las transferencias de derechos de emisión entre las entidades subnacionales participantes, o el establecimiento del Low Carbon Fuel Standard de carácter regional, con el objetivo de reducir las emisiones de GEI procedentes de los vehículos. Por otro lado, la intención de los promotores de este esquema de comercio de emisiones

\footnotetext{
${ }^{71}$ Regional Greenhouse Gas Initiative, Memorandum of Understanding, art. 2. F.1.a, recuperado el 25 diciembre $2010 \mathrm{de}$ http://rggi.org/docs/mou_12_20_05.pdf.

${ }^{72}$ RGGI CO2 Allowance Tracking System, recuperado el 25 diciembre 2010 de https://rggi-coats.org/ eats/rggi/.
} 
es vincularlo formalmente con el sistema de comercio de emisiones de la UE - un aspecto al que se hace referencia en el próximo apartado de este trabajo- y con el sistema de comercio de emisiones promovido por otra asociación norteamericana, la Western Climate Initiative ${ }^{73}$.

\subsection{Western Climate Initiative 74}

La Western Climate Initiative (en adelante WCI) es una asociación creada por los gobernadores de los estados de Washington, Oregón, Arizona, Nuevo México y California, mediante el Memorandum of Understanding de 26 de febrero de 200775. Posteriormente, las provincias canadienses de Québec, Ontario, Columbia Británica y Manitoba se sumaron al proyecto, junto con los estados de Utah y Montana en Estados Unidos. Además, hay otras 14 entidades que tienen un estatuto de observador: en Estados Unidos, Alaska, Colorado, Idaho, Kansas, Nevada y Wyoming; en Canadá, Nueva Escocia y Saskatchewan; y en México, Baja California, Chihuahua, Coahuila, Nuevo León, Sonora, y Tamaulipas.

El objetivo de la red se concretó en agosto de 2007, con el anuncio de una reducción de las emisiones del 15\% respecto de los niveles del año 2005, a lograr en 2020. El límite inicial se fija en la mejor estimación de las emisiones actuales entre 2012 y 2015, y se establece una reducción anual hasta 2020 que responde a un factor lineal.

El sistema "cap \& trade" 76 tiene que iniciarse en 2012, y se aplicará a las emisiones procedentes de la generación de energía eléctrica, a las grandes instalaciones industriales y comerciales y a parte de las emisiones procedentes del transporte de diesel

73 Una primera muestra de la colaboración entre estas asociaciones regionales ha cristalizado en la adopción, por parte de la Midwestern Greenhouse Gas Reduction Accord, la Regional Greenhouse Gas Initiative y la Western Climate Initiative, del documento Ensuring Offset Quality: Design and Implementation Criteria for a High-Quality Offset Program, en mayo de 2010, recuperado el 25 de diciembre de 2010 de http://www.midwesternaccord.org/News\%20Page/ThreeRegions_Offsets_Whitepaper\%2005_17_10.pdf.

74 Western Climate Initiative, recuperado el 25 diciembre 2010 de http:// www.westernclimateinitiative.org/.

75 Texto recuperado el 25 de diciembre 2010 de http:/www.ecy.wa.gov/climatechange/docs/ 07Mar_WesternRegionalClimateActionInitiative.pdf.

76 Design Recommendations for the WCI Regional Cap-and-Trade Program, September 23, 2008, texto recuperado el 25 de diciembre 2010 de http://www.westernclimateinitiative.org/component/remository/ func-startdown/15/. 
y de gas y del uso de combustibles fósiles en el sector residencial, comercial e industrial. A diferencia del anterior, pues, se trata de un programa de carácter global y multisectorial, que, cuando se haya implementado en su totalidad, cubrirá cerca del $90 \%$ de las emisiones de los estados y las provincias que participan en el mismo. Con respecto a la forma de asignación de los derechos de emisión, se dispone inicialmente la subasta para al menos el 10\% de los derechos, una cantidad que se incrementa hasta el $25 \%$ en 2020 .

La ejecución del programa se divide en dos fases. La primera se inicia en enero de 2012 e incluye las emisiones procedentes de la generación de electricidad, incluida su importación, la combustión en grandes instalaciones industriales y las emisiones procedentes de los procesos industriales que se puedan medir específicamente. La segunda fase empezará en 2015, cuando el programa se ampliará al transporte de combustibles y a la combustión en usos residenciales, comerciales e industriales. El sistema tendrá periodos de cumplimiento de tres años (2012-2014, 2015-2017 y 2018-2020). Por otro lado, la acción de la WCI se desarrolla paralelamente a la política de sus miembros en materia de lucha contra el cambio climático, incluidas las medidas fiscales. Así, se prevé expresamente que los socios "may use fiscal measures that contribute to achieving overall comparable GHG emission reductions and internalize the price of carbon as expected through the regional cap-and-trade program for transportation and residential/commercial fuels"77 y se prevé también que en un futuro se pueda integrar el sistema de mercado de emisión con la imposición de tasas CO2 en algunos estados o provincias $^{78}$.

Hasta ahora, las relaciones de la WCI se han producido esencialmente con otras iniciativas de su misma área geográfica, como la Regional Greenhouse Gas Initiative, el Midwest Greenhouse Gas Accord, la Southwest Climate Change Initiative ${ }^{79}$ y la West

\footnotetext{
77 Design Recommendations for the WCI Regional Cap-and-Trade Program, September 23, 2008, texto recuperado el 25 de diciembre 2010 de http://www.westernclimateinitiative.org/component/remository/ func-startdown $/ 15 /$.

78 Vid. sobre este punto, JOHNSTONE, N., The use of tradable permits in combination with other environmental policy instruments, OCDE, París, 2003.

${ }^{79}$ Creado en febrero de 2006 por Arizona y Nuevo México, en Southwest Climate Chanmge Initiative, recuperado el 25 de diciembre 2010 de http://www.nmclimatechange.us/ewebeditpro/items/ O117F8087.pdf.
} 
Coast Governors' Global Warming Initiative ${ }^{80}$. No obstante, el objetivo de la WCI es más ambicioso y prevé de manera expresa su posible vinculación con otros sistemas a nivel internacional, en particular con el sistema de comercio de derechos de emisión de la UE. Así se dispone en las Design recommendations que "The WCI Partner jurisdictions will seek bilateral and multilateral linkages with other governmentapproved cap-and-trade systems so that those allowances and allowances issued by WCI Partner jurisdictions would be fully fungible"81. También se prevé la compatibilidad del sistema con la aprobación de futuros sistemas de comercio de emisiones de GEH a nivel federal, sea en los Estados Unidos o en Canadá, por lo que se prevé que "In the event WCI issues allowances before a federal program in Canada or the United States, WCI Partner jurisdictions will work to ensure that those allowances are fully recognized and valued in the operation of a federal program" $" 2$.

\subsection{Midwestern Regional Greenhouse Gas Reduction Accord ${ }^{83}$}

El Midwestern Regional Greenhouse Gas Reduction Accord (en adelante MRGGRA) se adoptó en el marco del Midwestern Energy, Securty \& Climate Stewardship Summit el 15 de noviembre de 2007, mediante el acuerdo ${ }^{84}$ de los estados estadounidenses de Illinois, Iowa, Kansas, Michigan, Minnesota y Wisconsin, y la provincia canadiense de Manitoba, a los que se sumó posteriormente el estado de Misouri. Participan también en calidad de observadores los estados de Indiana, Ohio y Dakota del Sur, y la provincia canadiense de Ontario.

\footnotetext{
${ }^{80}$ Creado en Noviembre de 2004 por Washington, Oregón, y California; en West Coast Governors' Global Warming Initiative, recuperado el 25 de diciembre 2010 de http:/www.ef.org/westcoastclimate/.

81 En Design Recommendations for the WCI Regional Cap-and-Trade Program, September 23, 2008, recuperado el 25 de diciembre $2010 \mathrm{de}$ http://www.westernclimateinitiative.org/component/remository/ func-startdown $/ 15 /$.

82 Ibid.

83 En Midwestern Regional Greenhouse Gas Reduction Accord, recuperado el 25 de diciembre 2010 de http://www.midwesternaccord.org/.

84 En Midwestern Greenhouse Gas Accord 2007, recuperado el 25 de diciembre 2010 de http:// www.midwesternaccord.org/midwesterngreenhousegasreductionaccord.pdf.
} 
El acuerdo prevé para el año $2020^{85}$ unos objetivos de reducción de las emisiones de los GEI que se han cuantificado en un $20 \%$ respecto de los niveles de emisiones del año 2005. Este objetivo se incrementa hasta un $-80 \%$ para 2050 . Con respecto al establecimiento del programa de comercio de derechos de emisión, el MRGGRA ha adoptado en mayo de 2010 las directrices sobre las que se basa esta iniciativa ${ }^{86}$, si bien la primera fase del programa no se iniciará hasta doce meses después de que el sistema haya sido definitivamente adoptado entre las entidades participantes. En ellas se recomienda, por ejemplo, el establecimiento de un único sistema híbrido entre la subasta y la asignación gratuita, o la armonización de los mecanismos para salvaguardar el valor en el mercado de los derechos de emisión, pero se deja la mayoría de las cuestiones en manos de las entidades participantes.

Se prevé también la posibilidad de vinculación del sistema a otros mecanismos de comercio de emisiones o de proyectos de reducción. Así, se mencionan expresamente la RGGI y la WCI a nivel regional, y el sistema de comercio de derechos de emisión de la UE a nivel internacional, además de "Other mandatory GHG reduction programs as appropriate" 87 . Finalmente, para el caso de que en un futuro se acabe adoptando un sistema de comercio de emisiones a nivel federal, sea en Estados Unidos o en Canadá, paralelo al sistema del MRGGRA, se prevé expresamente que "the jurisdictions should work to ensure that those allowances are fully recognized and valued in the operation of a federal program." 88

\section{UN CASO ESPECIAL DE INTERVENCIÓN DE LAS ENTIDADES SUB- NACIONALES: LA VINCULACIÓN DE LOS SISTEMAS DE COMERCIO DE}

\footnotetext{
85 En Midwestern Greenhouse Gas Reduction Accord: Final Recommendations of the Advisory Group, recuperado el 25 de diciembre 2010 de

http://www.midwesternaccord.org/Accord_Final_Recommendations.pdf.

86 En Midwestern Regional Greenhouse Gas Reduction Accord, recuperado el 25 de diciembre 2010 de http://www.midwesternaccord.org/Accord_Final_Recommendations.pdf.

87 En Midwestern Regional Greenhouse Gas Reduction Accord, recuperado el 25 de diciembre 2010 de http://www.midwesternaccord.org/Accord_Final_Recommendations.pdf.

88 En Midwestern Regional Greenhouse Gas Reduction Accord, recuperado el 25 de diciembre 2010 de http://www.midwesternaccord.org/Accord_Final_Recommendations.pdf.
} 


\section{EMISIONES NORTEAMERICANOS CON EL SISTEMA DE COMERCIO DE EMISIONES DE LA UNIÓN EUROPEA}

La vinculación de los sistemas de comercio de emisiones de GEI permite promover la utilización de las unidades de uno de los sistemas en otros sistemas por parte de los actores participantes, a los efectos de que estos puedan acreditar al final del periodo de compromiso el cumplimiento con los objetivos de reducción que se les han sido asignados. Esta vinculación puede tener un carácter unilateral, cuando sólo uno de los sistemas acepta reconocer derechos de emisión procedentes de un sistema foráneo para acreditar el cumplimiento de los objetivos de reducción (es decir, las entidades del sistema A pueden comprar y utilizar derechos de emisión del sistema B pero no a la inversa); bilateral, cuando esta mención explícita y este reconocimiento se produce entre dos sistemas (por lo tanto, en cada sistema los derechos de emisión sirven para el cumplimiento de las obligaciones de reducción que se imponen); o multilateral, cuando esta posibilidad tiene lugar entre múltiples sistemas (un ejemplo de sistema totalmente multilateral es el de la vinculación de los sistemas nacionales dentro de la UE) ${ }^{89}$. También puede producirse directamente, de modo que se permite que los derechos de emisión puedan circular libremente dentro y entre los diferentes sistemas de comercio de derechos de emisión; o indirectamente, cuando por ejemplo, dos sistemas de comercio de derechos de emisión se vinculan a un tercero que genera créditos procedentes de la ejecución de proyectos de reducción que pueden después intercambiarse por derechos de emisión y aplicarse igualmente al cumplimiento de los objetivos de reducción ${ }^{90}$ (por ejemplo, si el sistema de la UE se vinculara al sistema de los Estados Unidos y al sistema de Australia, los desarrollos en los sistemas norteamericano y australiano tendrían un impacto uno en el otro aunque no estuvieran directamente vinculados entre ellos). De entrada, este tipo de vínculos entre sistemas de comercio de derechos de emisión crea una mayor diversidad de opciones de reducción,

\footnotetext{
89 Vid. STERK, W., MEHLING, M., TUERK, A., "Prospects of linking EU and US Emission Trading Schemes: Comparing the Western Climate Initiative, the Waxman-Markey and the Lieberman-Warner Proposals", Climate Strategies, April 2009, p. 3, en Ecologic Institute, recuperado el 25 de diciembre 2010 de http://ecologic.eu/3199.

90 Vid., FIELD-IEEP-WRI, Analysis of the legal and organizational issues arising in linking the EU Emissions Trading Scheme to other existing and emerging emissions trading schemes. Final Report, May 2008, recuperado el 25 de diciembre 2010 de http:/www.field.org.uk/files/Linking\%20emission $\% 20$ trading\%20schemes.pdf, p. 3 y ss.
} 
mejora la liquidez del mercado, permite una asignación más eficaz de los recursos y reduce los costes de la aplicación y el cumplimiento. Al mismo tiempo, la vinculación de sistemas de comercio de emisiones permite reducir ciertas distorsiones a la competencia ${ }^{91}$.

La vinculación de los diferentes sistemas de comercio de derechos de emisión de GEI era un objetivo que estaba presente en el sistema comunitario desde 2003. El artículo 25 de la Directiva 2003/87/CE, en su versión anterior a 2009, permitía la conclusión de acuerdos "con terceros países mencionados en el anexo B del Protocolo de Kyoto que hayan ratificado dicho Protocolo, a efectos de establecer el reconocimiento mutuo de los derechos de emisión entre el régimen comunitario y otros regímenes de comercio de derechos de emisión de gases de efecto invernadero"92. La Directiva 2004/101/CE ${ }^{93}$, por la que se vincula el sistema de comercio de emisión comunitario con los mecanismos de proyecto del PK, va más allá y requiere de la Comisión que examine la posibilidad de acuerdos con países que no han ratificado todavía el PK. Finalmente, el parágrafo 27 del artículo 1 de la Directiva 2009/29/CE ha incorporado la posibilidad de que estos acuerdos se puedan hacer "entre el régimen comunitario y otros regímenes obligatorios de comercio de derechos de emisión de gases de efecto invernadero compatibles que establezcan límites máximos de las emisiones en términos absolutos en cualquier otro país o entidad sub-federal o regional"94. Se dispone además que "Podrán celebrarse acuerdos no vinculantes con terceros países o entidades sub-federales o regionales para la coordinación técnica y administrativa en relación con los derechos de emisión en el régimen comunitario o en otros regímenes obligatorios de comercio de

91 STERK, W., MEHLING, M., TUERK, A., "Prospects of linking EU and US Emission Trading Schemes: Comparing the Western Climate Initiative, the Waxman-Markey and the Lieberman-Warner Proposals", Op.cit., p. 3; y STERK, W- BRAUN, M., HAUG, C.-KORITAROVA, K.-SCHOLTJEN, A.,"Ready to Link Up? Implication of design Differences for Linking Domestic Emissions Trading Schemes", Joint Emission Trading as Socio-Ecological Transformation, Cross section Project 4, JETSET Working Paper I/06, recuperado el 25 de diciembre 2010 de http://www.wupperinst.org/uploads/ tx_wibeitrag/ready-to-link-up.pdf, p. 12.

92 Como ha señalado WITT WIJNEN, hasta la modificación de la Directiva en el 2009, "everyone can trade Kyoto units; but when trade leads to a transfer of allowances, the transfer can be made as a general rule, only by Parties to the Kyoto Protocol", WITT WIJNEN,R., "Emission trading under Article 17 of the Kyoto Protocol", en FREESTONE,D.-STRECK,C. (ed), Legal Aspects of Implementing the Kyoto Protocol Mechanisms: Making Kyoto Work, Oxford, 2005, p. 412.

${ }^{93}$ DOUE L 338, de 13.11.2004.

${ }^{94}$ DOUE L 140, de 5.6.2009. 
derechos de emisión de gases de efecto invernadero con límites máximos de emisión en términos absolutos.". Por lo tanto, a diferencia de la Directiva de 2003, que dejaba fuera la posibilidad de vinculación del sistema comunitario con otras entidades que no fueran Estados o con terceros Estados que no hubieran ratificado el $\mathrm{PK}^{95}$, ahora la nueva Directiva permite esta vinculación no sólo con las autoridades centrales o federales de un tercer Estado sino también sub-nacionales. Hasta entonces, el principal debate se centraba en qué se podía vincular y cómo se podía vincular. Ahora se plantea también entre quién se puede producir esta vinculación. A pesar de que no se indica, como es natural, cuál es el nivel habilitado para concluir estos acuerdos, es curioso que sea justamente la norma comunitaria la que abre el camino a la participación de las entidades sub-nacionales y permite visualizar sus intereses en el contexto internacional. Es desde esta perspectiva que hay que examinar brevemente los retos que presenta la posible vinculación de los sistemas de comercio de derechos de emisión articulados por las entidades sub-nacionales norteamericanas mediante las redes transnacionales con el sistema de la UE.

\section{Las limitaciones materiales a la conclusión de acuerdos de vinculación de los sistemas de comercio de derechos de emisión}

Desde el punto de vista técnico debe garantizarse que el reconocimiento mutuo de los derechos de emisión foráneos no ponga en entredicho ni la eficacia de los regímenes previstos en los sistemas norteamericanos, ni la eficacia del sistema previsto en la Directiva europea 2003/87/CE.

Especialmente es necesario asegurar la fungibilidad de los derechos de emisión expedidos en unos y otros sistemas. Al respecto, existen algunos elementos en los que resulta esencial garantizar una mínima compatibilidad. Ello es así, por ejemplo, respecto de los mecanismos de supervisión y notificación de las emisiones por parte de los operadores de las instalaciones, los mecanismos para determinar el volumen de derechos que pueden ser expedidos por las terceras partes, y los períodos de validez de

\footnotetext{
95 Los terceros Estados incluidos en el anexo B y que han ratificado el PK son Noruega, Suiza, Canadá, Japón, Nueva Zelanda, Australia, Mónaco, Liechtenstein y la Federación Rusa. Otros dos Estados, actualmente inmersos en el proceso de adhesión a la UE también lo han hecho, Islandia y Croacia. El único que sigue sin haberlo ratificado es Estados Unidos.
} 
los derechos de emisión, puesto que en caso contrario el volumen de las emisiones futuras no sería controlable o manejable ${ }^{96}$.

Asimismo, debe evitarse que un tratamiento divergente de sectores semejantes (por ejemplo, el caso de las nucleares o de las hidroeléctricas) en ambos sistemas de comercio de emisiones estén causadas, justamente, por la vinculación de los sistemas de comercio de los derechos de emisión, puesto que podrían suscitarse cuestiones relativas a la competitividad de ambos sistemas. Otro aspecto importante es el reconocimiento de las unidades de emisión externas al propio sistema de comercio de emisiones, puesto que si una determinada unidad no se acepta en uno de los sistemas, los operadores económicos que se mueven en otro sistema que sí que las reconoce pueden igualmente venderlas a los primeros a los efectos del cumplimiento nacional de los objetivos de reducción. En este caso, el sistema que reconoce más restrictivamente los derechos de emisión foráneos tiene que adoptar medidas de adaptación, como la introducción de tasas de intercambio, lo que incrementa entonces los gastos de transacción ${ }^{97}$.

En cambio, hay otros elementos en los que la diversidad no resulta, en principio, un impedimento para la puesta en marcha del sistema de vinculación. A título de ejemplo, una cobertura distinta en lo que se refiere a los sectores o a los gases no debería implicar una incompatibilidad irresoluble desde el punto de vista institucional, como tampoco tiene porque disminuir la efectividad ambiental de un sistema de comercio de emisiones integrado. Asimismo, las diferencias respecto a la forma en que se asignan los derechos de emisión a los operadores económicos tampoco debería tener impacto desde el punto de vista de la efectividad ambiental, puesto que esto se determina solamente en función del límite máximo que se establece (si bien sí que tiene una clara incidencia económica en la medida en que la asignación gratuita se puede asimilar a una subvención).

96 Vid. FIELD-IEEP-WRI, Analysis of the legal and organizational issues arising in linking the EU Emissions Trading Scheme to other existing and emerging emissions trading schemes. Op.Cit, p. 44; BARON, R.-BYGRAVE, S., Towards International Emissions Trading: Design implications for linkages, OECD, Paris, 2002, en OECD-IEA Information Paper, recuperado el 25 de diciembre 2010 de http:// www.iea.org/papers/2002/linkages.pdf, p. 8 y ss.

97 STERK, W., MEHLING, M., TUERK, A., Prospects of linking EU and US Emissions Trading Schemes: Comparing the Western Climate Initiative, the Waxman-Markey and the Lieberman-Warner Proposals, Op.Cit., 
Por estas razones, al articular esta vinculación entre diferentes sistemas de comercio de derechos de emisión hay algunos elementos que deben considerarse muy cuidadosamente. En particular debe salvaguardarse la integridad ambiental del sistema de comercio de emisiones, es decir, hay que evitar que se generen reducciones menores que las que se producirían sin vincular los sistemas; su integridad institucional, y por lo tanto, la compatibilidad de las estructuras y los elementos que caracterizan el sistema; su eficiencia económica, de tal manera que la vinculación con otros sistemas de comercio mejore la relación coste-beneficio respecto al funcionamiento aislado de cada uno de ellos; $y$, finalmente, la equidad del sistema respecto a la igualdad de condiciones entre sus participantes ${ }^{98}$.

\section{Las limitaciones formales a la conclusión de acuerdos de vinculación de los sistemas de comercio de derechos de emisión}

Como se ha indicado, tanto la RGGI, como la WCI y el MRGGRA en Norteamérica prevén de manera expresa la posibilidad de vincular, mediante un acuerdo internacional, sendos sistemas de comercio de derechos de emisión con el sistema de comercio de derechos de emisión de la UE, una eventualidad que a su vez contempla la Directiva 2003/87/CE modificada por la Directiva 2009/29/CE. Ello sin perjuicio de que en el caso de Estados Unidos y Canadá los efectos de esta vinculación puedan ser diferentes si finalmente sólo existe un sistema de alcance federal en cada uno de los dos países, o si por el contrario éstos deben convivir con sistemas estatales o provinciales.

No obstante, la UE difícilmente podrá concertar convencionalmente esta vinculación en el caso de que finalmente no se establezca un sistema federal de comercio de derechos de emisión en los Estados Unidos y en Canadá- con los sistemas previstos en las asociaciones mencionadas. Como ya se ha dicho, éstas son redes transnacionales que como tales carecen de personalidad jurídica internacional, y que por lo tanto difícilmente pueden ser parte en un tratado internacional. Ante esta situación

\footnotetext{
98 FIELD-IEEP-WRI, Analysis of the legal and organizational issues arising in linking the EU Emissions Trading Scheme to other existing and emerging emissions trading schemes. Op.Cit., p. 4 y p. 51; y STERK,W., MEHLING,M., TUERK,A., Prospects of linking EU and US Emission Trading Schemes: Comparing the Western Climate Initiative, the Waxman-Markey and the Lieberman-Warner proposals, Op.Cit., p.3; STERK, W., BRAUN, M., HAUG, C., KORITAROVA, K., SCHOLTJEN, A.,'Ready to Link Up? Implication of design Differences for Linking Domestic Emissions Trading Schemes", Op. Cit. p. 14 y ss.
} 
probablemente será necesario recurrir a otros mecanismos alternativos para realizar dicha vinculación, como por ejemplo, un acuerdo informal entre los gobiernos implicados, una declaración política conjunta o un memorándum de entendimiento, todos ellos sin embargo instrumentos de una alcance más político que jurídico. También cabe vincular los sistemas de comercio mediante instrumentos de derecho privado, por ejemplo a través de la creación de un sistema de conversión de las unidades y de los derechos de emisión.

Asimismo, esta incapacidad para concluir los acuerdos internacionales necesarios para vincular los distintos sistemas de comercio de derechos de emisión no afecta solamente a estas asociaciones sino que está también presente en lo que respecta a los estados y a las provincias en Estados Unidos y Canadá.

En Estados Unidos, conforme al artículo I.10 de la Constitución "No State shall enter into any Treaty, Alliance, or Confederation.". Igualmente se indica que "No State shall, without the Consent of Congress, (...) enter into any Agreement or Compact with another State, or with a foreign Power (...).” En esencia, esta disposición niega a los estados federados la personalidad jurídica internacional y limita claramente su posibilidad de participar en las relaciones internacionales ${ }^{99}$, a menos que exista el consentimiento expreso del Congreso ("agreement or compact clause") ${ }^{100}$.

No obstante, de acuerdo con los Restatements del Americal Law Institute, "By analogy with inter-State compacts, a State compact with a foreign power requires Congressional consent only if the compact tends "to the increase of political power in the States which may encroach upon or interfere with the just supremacy of the United (...). In general, agreements involving local transborder issues, such as agreements to curb a source of

99 Vid. HENKIN, L. Foreign Affairs and the US Constitution, Oxford University Press, Oxford-NY, 2002; GOLDSMITH, J.L., "New Formalisms in United States Foreign Relations", en University of Colorado Law Review, núm. 70, 1999, p.1395; YOO, J.C., "Globalism and the Constitution: Treaties, NonselfExecution, and the Original Understanding", en Columbia Law Review, núm. 99, 1999, p. 1956; GOLOVE, D.M., "Treaty-Making and the Nation: The Historical Foundations of the Nationalist Conception of the Treaty Power”, en Michigan Law Review, núm. 98, 2000, p. 1075.

100 "What distinguishes a treaty, which a State cannot make at all, from an agreement or compact, which it can make with Congressional consent, has not been determined. That would probably be deemed a political decision. Hence, if Congress consented to a State agreement with a foreign power, courts would not be likely to find that it was a "treaty" for which Congressional consent was unavailing", REST 3d FOREL § 302, Restatement (Third) of Foreign Relations Law \& 302 (1987), Restatement of the Law Foreign Relations Law of the United States, Part III. International Agreements, Chapter 1. International Agreements: Definition, Nature, And Scope, § 302. Scope Of International Agreements: Law Of The United States. 
pollution (...) have been considered not to require Congressional consent. Such agreements are not international agreements under the criteria stated in $\S 301(1)$, but other State compacts might be"101. Se apunta pues la posibilidad de que en ciertos supuestos se pudiera adoptar un determinado tipo de acuerdos incluso sin el consentimiento del Congreso, lo que supondría una mayor presencia de los estados en la escena internacional ${ }^{102}$.

Ahora bien, ésta es una situación sumamente improbable en la práctica por el propio funcionamiento del sistema americano. De hecho, parte de la doctrina sostiene una intervención inexistente de los estados en el marco de las relaciones internacionales, hasta el punto de considerar que "At the end of the twentieth century as at the end of the eighteenth, as regards U.S. foreign relations, the states do not exist" ${ }^{\prime 103}$ y constatar que "although there is much debate about the legitimacy of federal common law generally a remarkable consensus about the legitimacy of the federal common law of foreign relations (...) Foreign relations is a special case because it is viewed as the exclusive prerogative of the federal government."104. Esta limitación respecto a la capacidad de los estados permite cuestionar la posibilidad de que las iniciativas presentadas puedan vincularse jurídicamente a un sistema de comercio de derechos de emisión como el de la UE.

\footnotetext{
${ }^{101}$ REST 3d FOREL $\S 302$, Restatement (Third) of Foreign Relations Law $\$ 302$ (1987), Restatement of the Law - Foreign Relations Law of the United States, Part III. International Agreements, Chapter 1. International Agreements: Definition, Nature, And Scope, § 302. Scope Of International Agreements: Law Of The United States.

${ }^{102}$ Vid. SWAINE, E.T., "Does Federalism Constrain the Treaty Power?”, en Columbia Law Review, núm. 103, 2003, p. 403.

${ }^{103}$ HENKIN, L. Foreign Affairs and the US Constitution, Op.Cit., p. 150.

104 GOLDSMITH, J.L., "Federal Courts,Foreign Affairs, and Federalism”, en Virginia Law Review, núm. 83,1997, p. 1617, en p. 1632.
} 
El caso de Canadá es más complicado ${ }^{105}$. De entrada, la negociación y la celebración de los tratados internacionales corresponde al gobierno federal ${ }^{106}$. El gobierno de Ottawa y la mayoría de la doctrina anglosajona ha sostenido reiteradamente la exclusividad de la competencia federal en materia de relaciones exteriores ${ }^{107}$. Desde esta perspectiva, ni las provincias canadienses constituyen sujetos de derecho internacional, puesto que no tienen el atributo externo que caracteriza la soberanía, ni la Constitución canadiense les reconoce la capacidad de ser titular de derechos y obligaciones en el ámbito internacional. La jurisprudencia del Tribunal Supremo ha ido también en esta misma línea y ha negado hasta ahora la capacidad de las provincias para actuar en el ámbito internacional, tal como se desprende claramente de la decisión del Tribunal Supremo de 1984 en el asunto Reference re Newfoundland Continental Shelf ${ }^{108}$.

Parte de la doctrina francófona defiende la tesis contraria y por lo tanto la capacidad de las provincias para negociar y concluir acuerdos internacionales cuando se trata de materias que tienen atribuidas constitucionalmente de manera exclusiva, puesto que de lo contrario se produciría un importante vacío jurídico ${ }^{109}$. Para este sector doctrinal, se puede fundamentar el treaty making power de las provincias en varias decisiones

105 Vid. GOTLIEB, A.E, Canadian Treaty Making, Butterworths, Toronto, 1968; KINDRED,H.M. (ed.), International Law: Chiefly as Interpreted and Applied in Canada, Emond Montgomery, $7^{\text {th }}$ ed., Toronto, 2006; DE MESTRAL, A., "L'evolution des rapports entre le droit canadien et le droit international un demi-siècle après l'Affaire des Conventions internationales du travail", en Canadian Yearbook of International Law, núm. 25, 1987, p. 301; JACOMY-MILLETE, A.M., Treaty Law in Canada, University of Ottawa Press, Ottawa, 1975.

106 Vid. HARRINGTON, J., "Redressing the Democratic Deficit in Treaty Law-Making: (Re-) Establishing a Role for Parliament”, en McGill Law Journal, núm. 50, 2005, p. 465; CYR,H., Canadian Federalism and Treaty Powers: Organic Constitutionalism at Work, Peter Lang, Brussels, 2008.

107 Vid. GOTLIEB, A.E., Canadian Treaty Making, Op.Cit, p.27 y ss.; MACDONALD,R.St.J., "International Treaty Law and the Domestic Law of Canada", en Delhousie Law Journal, núm. 2, 1975, p.307; MORRIS, G.L., "Canadian Federalism and International Law", en MACDONALD,R.St.J.MORRIS,G.L.-JOHNSTON,D.M. (ed.), Canadian Perspectives on International Law and Organization, University of Toronto Press, Toronto-Buffalo, 1974, p. 55., BOULAC, S., "The Canadian Federal Constitutional Framework and the Implementation of the Kyoto Protocol", en COSTI,A.-SAGE,Y.L. (ed.), Essays on Environmental Law in the Pacific. International and Comparative Perspectives, RJP Special Issue, Vol.. V, New Zealand Association for Comparative Law and Association de Législation Comparée des Pays du Pacifique, 2005, p. 125.

108 Reference re Newfoundland Continental Shelf, [1984] 1 S.C.R. 86, p. 103, a http:// csc.lexum.umontreal.ca/en/1984/1984scr1-86/1984scr1-86.html

109 Vid. MORIN, J.-Y., "La personalité internationale du Quebec", en Revue Québecoise de Droit International, núm. 1, 1984, p.199; MORIN, J.-Y., "Le féderalisme canadien: un dilemme en matière de traités", en Annuaire Canadien de Droit International, 1965, p. 130; PAQUIN,S.-BEAUDOIN,L., (dir.), Les relations internationales du Québec depuis la doctrine Gérin-Lajoie (1965-2005): le prolongement externe des compétences internes, Presses de l'Université Laval, Québec, 2006, p.49. 
adoptadas por el Comité Judicial del Consejo Privado de la Cámara de los Lores a comienzos de siglo $\mathrm{XX}^{110}$, en las cuales se sostiene que la Constitución se limita a conferir al gobierno federal la administración de los asuntos en los cuales las provincias tienen un interés común, sin perjuicio de su independencia y que por esto estas ejercen las prerrogativas de la Corona en el ámbito de las competencias provinciales, que comprende también el poder de contraer obligaciones internacionales.

Esta cuestión se plantea desde entonces de manera casi permanente en Québec, y de hecho los sucesivos gobiernos quebequenses han hecho uso reiterado de esta doctrina equiparando la prolongación de las competencias internas y externas. La doctrina de Gérin-Lajoie, en definitiva, reconoce claramente la capacidad del Québec para negociar y concluir tratados internacionales en aquellos ámbitos que afecten a sus competencias $^{111}$, una opción que sus defensores consideran lógica dado que de hecho corresponde a la provincia la aplicación de los mismos tratados internacionales en los ámbitos de sus competencias ${ }^{112}$.

\section{CONCLUSIONES FINALES}

En un primer orden de conclusiones y en lo que se refiere al desarrollo normativo del régimen internacional del cambio climático, debe constatarse la relevancia de la participación de las entidades sub-nacionales en Norteamérica A diferencia de lo que

\footnotetext{
110 Re Liquidators of the Maritime Bank v. The Receiver-General of the New Brunswick, Law Reports. Appeal Cases, 1892, p. 437, y Re Bonanza Creek Gold Mining Company Ltd. v. The King, Law Reports. Appeal Cases., 1916, p. 566.

${ }^{111}$ MICHAUD, N., « La doctrine Gérin-Lajoie », en PAQUIN,S.- BEAUDOIN,L. (dir.), Histoire des Relations Internationales du Québec, VLB éditeur, Montréal, 2006, p. 263; TURP,D., " La doctrine Gérin-Lajoie et l'émergence d'un droit québécois des relations internationales» en PAQUIN,S.BEAUDOIN, L. (dir.), Histoire des Relations Internationales du Québec, Op.Cit., p.49, en p. 57.
}

112 Así, en el 2002 la Asamblea Nacional quebequesa adoptó unánimemente la Loi modifiant la loi sur le ministère des Relations internationales et d'autres dispositions législatives (projet de loi 52, L.Q. 2002, c. 8 ), por la que se exigía la aprobación por Québec de todo tratado internacional concluido por Canadá que afectara a sus competencias. Siete años después, el Bloque Quebequés ha presentado ante la Cámara de los Comunes de Canadá del Proyecto de ley núm. C-486, relativo a la negociación y a la conclusión de los tratados internacionales que retoma el mismo planteamiento. En particular, el nuevo proyecto de Ley compromete al gobierno federal a pactar, especialmente con Québec, el mandato confiado al ejecutivo canadiense cada vez que deba negociarse un acuerdo internacional que pueda afectar una materia en la que es competente la provincia, además de permitir la negociación directa y la conclusión de los acuerdos internacionales por Québec cuando estos afecten a sus competencias exclusivas. Vid. Projet de Loi C-486, Chambre des Communs, Deuxième session, quarantième Parlement, 57-58 Elizabeth II, 2009; Première lecture le 2 décembre 2009, en Parlement du Canada, LEGISinfo, recuperado el 25 de diciembre 2010 de http://www2.parl.gc.ca/sites/lop/legisinfo/index.asp?Language=F\&query=5972\&List=toc\&Session=22. 
ocurre en la UE, en el caso norteamericano la intervención de las entidades subnacionales en materia de lucha contra el cambio climático ha tenido y está teniendo un cariz totalmente diferente desde varias perspectivas. De entrada, a pesar de que no puede ignorarse la estrecha vinculación existente entre la política de cambio climático en Estados Unidos y en Canadá, por lo menos en lo que se refiere a las responsabilidades de los gobiernos federales, los instrumentos regionales no han facilitado la articulación de una política común. Por otro lado, mientras que en Estados Unidos se ha querido priorizar la autonomía, el enfoque regulatorio múltiple y las interacciones entre las jurisdicciones federal y estatal en materia de medio ambiente, en el caso de Canadá hay importantes limitaciones a la intervención federal y las provincias no han dejado nunca de reclamar el ejercicio concurrente de esta competencia al margen de que la Constitución prevea el ejercicio de las competencias en base a su exclusividad. De todas maneras, la intervención de las entidades subnacionales es en ambos casos indispensable para garantizar el cumplimiento de los compromisos internacionales, y en el supuesto que ahora nos ocupa, del PK; muy especialmente, los estados estadounidenses y las provincias canadienses están presentes en la elaboración y la aprobación de la normativa relativa al régimen de comercio de emisiones de GEI.

Puesto que ni Estados Unidos ni Canadá disponen todavía de un marco regulatorio de alcance federal en materia de reducción y limitación de las emisiones de GEI, la articulación de los regímenes de comercio de derechos de emisión de GEI en Norteamérica se enmarca hasta ahora en un contexto de una amplia descentralización. Es más, ha estado principalmente a cargo de las entidades sub-nacionales, que han desarrollado importantes actuaciones para poder disponer de instrumentos para el cumplimiento del PK, bien de manera unilateral, bien mediante la cooperación en el marco de redes de carácter transnacional como la Western Climate Initiative, la Regional Greenhouse Gas Initiative, y el Midwest Greenhouse Gas Reduction Accord.

En definitiva, la lucha contra el cambio climático se ha caracterizado en los últimos años en Norteamérica por el carácter sub-nacional de la mayoría de estas medidas y por la coordinación de las políticas sub-nacionales a través de redes transnacionales mencionadas. En la medida en que estas acciones cubren cerca de 2/3 del total de las 
emisiones de GEI de Norteamérica, la cuestión que entonces se plantea, y que hoy por hoy todavía no se ha respondido, es cómo esta situación afectará la relación entre los estados y las provincias parte en estas redes transnacionales y los gobiernos federales, respectivos y hasta qué punto el desarrollo de un sistema de comercio de derechos de emisiones de alcance federal es todavía relevante ${ }^{113}$.

En un segundo orden de consideraciones, cabe referirse a algunos de los retos que conlleva la eventual vinculación de los sistemas de comercio de derechos de emisión que en un futuro próximo pueden operar en Norteamérica con el sistema de la UE. Es en este contexto que cabe valorar la posibilidad que ofrece la Directiva 2003/87/CE modificada por la Directiva 2009/29/CE. De una parte, la reducción de emisiones de GEI en la UE a partir del periodo que se inicia en 2013 requerirá que los operadores económicos puedan solicitar el reconocimiento de los derechos de emisión entre el sistema de la UE y los diversos regímenes con quién ésta se vincule. De la otra, no obstante, la normativa de la UE no prevé un reconocimiento automático de los derechos de emisión obtenidos mediante la participación en otros sistemas, sino que lo supedita a la conclusión de futuros acuerdos internacionales con terceros Estados, entidades subnacionales o entidades regionales.

Más allá de las dificultades que se pueden presentar en relación con los aspectos más técnicos del reconocimiento de los derechos de emisión, la formalización jurídica del vínculo entre diferentes sistemas afronta el obstáculo importante de la falta de subjetividad internacional de las redes transnacionales, por un lado, y de las entidades sub-nacionales por el otro, con lo que difícilmente podrán firmarse los acuerdos necesarios con la UE para permitir el reconocimiento de los derechos de emisión que puedan generarse en ambos sistemas. No obstante, cabe considerar que si bien el recurso a instrumentos clásicos como el acuerdo internacional sin duda sigue siendo más eficaz y el que aporta un plus de transparencia en el establecimiento y la determinación del alcance de las obligaciones de los Estados y los organizaciones internacionales, pueden también contemplarse otras posibles formas para vincular el

\footnotetext{
113 Vid. PAPY, J., La conséquence de la participation des provinces canadiennes à la WCI : Un régime fédéral d'échange d'emissions est-il encore pertinent?. Presentación en el Colloque Fédéralisme, environnement et intégration régionale. Regards croisés sur la Belgique et le Canada, 27-28 de mayo de 2010, Bruselas, Bélgica.
} 
sistema de comercio de derechos de emisión de GEI de la UE con los sistemas que operan en el área norteamericana de manera que permita el reconocimiento de los derechos de emisión. Esto puede hacerse, por ejemplo a través de la creación de un marco de referencia para los dos sistemas mediante la adopción de acuerdos políticos, y por lo tanto, sin carácter vinculante, en los que ambas partes se comprometan a adaptar la normativa propia a las exigencias que implique su vinculación con otro sistema.

\section{BIBLIOGRAFÍA CITADA}

ADELMAN, D.E., ENGEL, K.H., "Adaptive Federalism: The Case Against Reallocating Environmental Regulatory Authority", en Arizona Legal Studies, Discussion Paper No. 07-23, September 2007, en University of Arizona, recuperado el 25 de diciembre 2010 de http://www.law.arizona.edu/faculty/FacultyPubs/Documents/ Adelman/ALS07-23.pdf.

AHDIEH, R.B., “Asuntos exteriores, derecho internacional y el nuevo federalismo: Lecciones derivadas de la coordinación", en Teoría y realidad constitucional, núm. 24, 2009, p. 109.

BACHELDER, A., "Using Credit Trading to Reduce Greenhouse Gas Emissions", en Environmental Law \& Practice, núm. 9, 2000, p. 281.

BARTON, P., "Economic Instruments and the Kyoto Protocol: Can Parliament Implement Emissions Trading Without Provincial Co-operation?", en Alberta Law Review, núm. 2, 2002, p. 417.

BEAUDOIN, G.A., « La protection de l'environnement et ses implications en droit constitutionnel», en McGill Law Journal, núm. 23, 1977, p. 207.

BEAULAC, S., "National Application of International Law : The Statutory Interpretation Perspective", en Canadian Yearbook of International Law, núm. 41, 2004, p. 225. 
BOULAC, S., "The Canadian Federal Constitutional Framework and the Implementation of the Kyoto Protocol", en COSTI,A.-SAGE,Y.L. (ed.), Essays on Environmental Law in the Pacific. International and Comparative Perspectives, RJP Special Issue, Vol.. V, New Zealand Association for Comparative Law and Association de Législation Comparée des Pays du Pacifique, 2005, p. 125.

BENIDICKSON, J., Environmental Law, $3^{\text {rd }}$ edition, Irwin Law, Toronto, 2009

CALABRESI, S.G., "A Government of Limited and Enumerated Powers: In Defense of United status v. López”, en Michigan Law Review, núm. 94, 1995, p. 752.

CASTELLA ANDREU, J.M., “Tribunal Supremo y cambios en el federalismo de Estados Unidos”, en Teoría y realidad constitucional, núm. 24, 2009, p. 491.

CHALIFOUR, N., "Making Federalism Work for Climate Change: Canada's Division of Powers over Carbon Taxes", en National Journal of Constitutional Law, núm 22, 2008, p. 1.

COHEN, M., "Canada and the International Legal Order: An Inside Perspective", en MACDONALD, R.St.J., MORRIS, G.L., JOHNSTON, D.M., Canadian Perspectives on International Law and Organization (ed.), University of Toronto Press, Toronto, 1974, p.3.

CYR, H., Canadian Federalism and Treaty Powers: Organic Constitutionalism at Work, Peter Lang, Brussels, 2008 .

DEIMANN, S., "R. v. Hydro-Québec: Federal Environmental Regulation as Criminal Law”, en McGill Law Journal, núm. 43, 1998, p. 923.

DE MESTRAL, A., FOX-DECENT, E., “ Rethinking the Relationship Between International and Domestic Law”, en McGill Law Journal, núm. 53, 2008, p. 573.

DE MESTRAL, A., “L'evolution des rapports entre le droit canadien et le droit international un demi-siècle après l'Affaire des Conventions internationales du travail », en Canadian Yearbook of International Law, núm. 25, 1987, p. 301.

DERNBACH, J. "Moving the Climate Debate from Models to Proposed Legislation: Lessons from State Experience", en Environmental Law Report, núm. 30, 2000, p. 10.933. 
DERNBACH, J.C., MCKINSTRY, R.B., PETERSON, T.D., Making the States Full Partners in a National Climate Change Effort: A Necessary Element for Sustainable Economic Development, Paper presented at 2nd UNITAR'-Yale Conference on Environmental Governance and Democracy, 17-19 September, New Haven, USA, recuperado el 25 de diciembre 2010 de http:/conference.unitar.org/yale/sites/ conference.unitar.org.yale/files/Paper_Dernbach.pdf.

ESTY, D., “Toward Optimal Environmental Governance”, en New York University Law Review, núm.74, 1999, p. 1495.

FABER, A., "The Constitution's Forgotten Cover Setter: An Essay on the New Federalism and the Original Understanding”, en Michigan Law Review, núm. 94, 1995, p. 610 .

FEREJOHN, J., WEINGAST, B.R. (ed.), The New Federalism. Can the States be Trusted?, Hoover Institution Press-Stanford University, Stanford, 1997.

FIELD-IEEP-WRI, Analysis of the legal and organizational issues arising in linking the EU Emissions Trading Scheme to other existing and emerging emissions trading schemes. Final Report, May 2008, recuperado el 25 de diciembre 2010 de http:// www.field.org.uk/files/Linking\%20emission\%20trading\%20schemes.pdf.

GERRARD, M.D. (Ed.) Global Climate Change and US Law, American Bar Association, 2007, recuperado el 25 de diciembre de 2010 de http:/www.abanet.org/ abapubs/globalclimate/docs/50States_Survey_ThruNov09final.pdf.

GIBSON, D. "Constitutional Jurisdiction over Environmental Management in Canada", en University of Toronto Law Journal, núm. 23, 1973, p. 54.

GOLDSMITH, J.L., "New Formalisms in United States Foreign Relations", en University of Colorado Law Review, núm. 70, 1999, p. 1395.

GOLDSMITH, J.L., "Federal Courts, Foreign Affairs, and Federalism”, en Virginia Law Review, núm. 83, 1997, p. 1617. 
GOLOVE, D.M., "Treaty-Making and the Nation: The Historical Foundations of the Nationalist Conception of the Treaty Power”, en Michigan Law Review, núm.98, 2000, p. 1075.

GOTLIEB, A.E, Canadian Treaty Making, Butterworths, Toronto, 1968

GRAGLIA, L.A., "United States v. Lopez: Judicial Review Ander the Comerse Clause”, en Texas Law Review, núm. 74, 1995, p. 720.

HARRINGTON,J., "Redressing the Democratic Deficit in Treaty Law-Making: (Re-) Establishing a Role for Parliament”, en McGill Law Journal, núm. 50, 2005, p. 465.

HENKIN, L. Foreign Affairs and the US Constitution, Oxford University Press, Oxford-NY, 2002

JACOMY-MILlETE, A.M., Treaty Law in Canada, University of Ottawa Press, Ottawa, 1975.

JOHNSTONE, N., The use of tradable permits in combination with other environmental policy instruments, OCDE, París, 2003.

KINDRED, H.M. (ed.), International Law: Chiefly as Interpreted and Applied in Canada, Emond Montgomery, $7^{\text {th }}$ ed., Toronto, 2006

LECLAIR, J., «Aperçu des virtualités de la compétence fédérale en droit criminel dans le contexte de la protection de l'environnement», en Revue Générale de Droit, núm. 27, 1996, p. 137.

MACDONALD, R. St.J., "International Treaty Law and the Domestic Law of Canada", en Delhousie Law Journal, núm. 2, 1975, p.307.

MORIN, J.-Y., "La personalité internationale du Quebec", en Revue Québecoise de Droit International, núm. 1, 1984, p.199.

MORIN, J.-Y., "Le féderalisme canadien: un dilemme en matière de traités", en Annuaire Canadien de Droit International, 1965, p. 130.

MORRIS, G.L., "Canadian Federalism and International Law", en MACDONALD,R.St.J.-MORRIS,G.L.-JOHNSTON,D.M. (ed.), Canadian Perspectives 
on International Law and Organization, University of Toronto Press, Toronto-Buffalo, 1974, p. 55.

PAPY, J., La conséquence de la participation des provinces canadiennes à la WCI : Un régime fédéral d'échange d'emissions est-il encore pertinent?. Presentación en el Colloque Fédéralisme, environnement et intégration régionale. Regards croisés sur la Belgique et le Canada, 27-28 de Mayo de 2010, Bruselas, Bélgica.

PAQUIN, S., BEAUDOIN,L., (dir.), Les relations internationales du Québec depuis la doctrine Gérin-Lajoie (1965-2005): le prolongement externe des compétences internes, Presses de l’Université Laval, Québec, 2006.

PAQUIN,S.- BEAUDOIN,L. (dir.), Histoire des Relations Internationales du Québec, VLB éditeur, Montréal, 2006.

PATENAUDE, M., “L'interprétation du partage des compétences à l'heure du libreéchange", en Revue de Droit de l'Université de Sherbrooke., núm. 21, 1990.

PERCIVAL, R., "Environmental Law in the Twenty-First Century", en Virginia Environmental Law Journal, núm. 25, 2007, p. 1.

RABE, B., "North American Federalism and Climate Change Policy: American State and Canadian Provincial Policy Development”, en Widener Law Journal, núm.14, 2004, p. 121.

RAUSTIALA, K., “The Architecture of International Cooperation: Transgovernmental Networks and the Future of International Law", en Virginia Journal of International Law, núm. 43, 2002, p. 1.

REY MARTÍNEZ, F., “United States v. Lopez y el nuevo federalismo americano”, en Revista Española de Derecho Constitucional, núm. 51, 1997, p. 273.

ROLAND-HOLST, D., Energy Efficiency, Innovation, and Job Creation in California; University of California, 2008, recuperado el 25 de diciembre 2010 de http:// are.berkeley.edu/ dwrh/CERES_Web/Docs/UCB\%20Energy\%20Innovation\%20and \%20Job\%20Creation\%2010-20-08.pdf. 
ROLFE, C., Turning Down the Heat: Emissions Trading and Canadian Implementation of the Kyoto Protocol, West Coast Environmental Law Research Foundation, Vancouver, 1998.

SCHRAMM, D.P., "Federal Midwife: Assisting the States in the Birth of a National Greenhouse Gas Cap-and-Trade Program", en Tulane Environmental Law Journal, núm. 22, 2008, p. 61.

SIMPSON, J., JACCARD,M.-RIVERS,N., Hot Air: Meeting Canada's Climate Change Challenge, McClelland \& Stewart Ltd., Toronto, 2007, p. 91.

SMITH, H.A., "Political parties and Canadian climate change policy", en International Journal, núm. 64, 2008-2009, p. 47.

SMITH, J.,VOGEL, J., CRUCE, T., SEIDEL, S., HOLSINGER, Adapting to Climate Change. A Call for Federal Leadership, Pew Center on Global Climate Change- The Rockefeller Foundation, April 2010, recuperado el 25 de diciembre 2010 de http:// www.pewclimate.org/docUploads/adaptation-federal-leadership.pdf.

STERK, W., MEHLING, M., TUERK, A., "Prospects of linking EU and US Emission Trading Schemes: Comparing the Western Climate Initiative, the Waxman-Markey and the Lieberman-Warner Proposals", Climate Strategies, April 2009, en Ecologic Institute, recuperado el 25 de diciembre 2010 de http://ecologic.eu/3199.

STERK, W., BRAUN, M., HAUG, C., KORITAROVA, K., SCHOLTJEN, A.,'Ready to Link Up? Implication of design Differences for Linking Domestic Emissions Trading Schemes", Joint Emission Trading as Socio-Ecological Transformation, Cross section Project 4, JET-SET Working Paper I/06, recuperado el 25 de diciembre 2010 de http:// www.wupperinst.org/uploads/tx_wibeitrag/ready-to-link-up.pdf.

STEWART, R.B., "Pyramids of Sacrifice? Problems of Federalism in Mandating State Implementations of National Environmental policy", en Yale Law Journal, núm. 6, 1977, p. 1196.

SWAINE, E.T., "Does Federalism Constrain the Treaty Power?", en Columbia Law Review, núm. 103, 2003, p. 403. 
TARLOK, D., "The Influence of International Environmental Law on United States Pollution Control Law”, en Vermont Law Review, núm. 21, 1997, p. 759.

TRUDEAU, H., "Le fédéralisme canadien et la protection de l'environnement", en Souveraineté et intégration, Actes du Colloque conjoint des Facultés de Droit de l'Université de Poitiers et de l'Université de Montréal, Université de Montréal, Ed. Thémis, 1993.

TRUDEAU, H., LALONDE, S., "La Mise en Oeuvre du Protocole de Kyoto au Canada: Concertation ou Coercition”, en Revue générale de droit, núm. 34, 2004, p. 141.

VAN ERT, G., "Using Treaties in Canadian Courts", en Canadian Yearbook of International Law, núm. 38, 2000, p. 3.

WAKUlAT, R., Canada's 2007 Climate Change Action Plan: Plan of Action or More Hot Air?, University of Toronto, 2008, reecuperado el 25 de diciembre 2010 de http:// www.cba.org/CBA/newsletters/pdf/10_08_env-essay.pdf.

WIENER, J.B., "Think Globally, Act Globally: The Limits of Local Climate Policies", en University of Pennsylvania Law Review, núm. 155, 2007, p. 107.

WITT WIJNEN, R., "Emission trading under Article 17 of the Kyoto Protocol", en FREESTONE,D.-STRECK,C. (ed), Legal Aspects of Implementing the Kyoto Protocol Mechanisms: Making Kyoto Work, Oxford, 2005, p. 412.

YOO, J.C., "Globalism and the Constitution: Treaties, Nonself-Execution, and the Original Understanding”, en Columbia Law Review, núm. 99, 1999, p. 1956. 